Review Article

\title{
Honey bee survival mechanisms against the parasite Varroa destructor: a systematic review of phenotypic and genomic research efforts
}

\author{
Fanny Mondet ${ }^{\mathrm{a}, 1, *}$, Alexis Beaurepaire ${ }^{\mathrm{a}, \mathrm{b}, 1}$, Alison McAfee $^{\mathrm{c}}$, Barbara Locke $^{\mathrm{d}}$, Cédric Alaux $^{\mathrm{a}}$, \\ Solene Blanchard ${ }^{a}$, Bob Danka ${ }^{\mathrm{e}}$, Yves Le Conte ${ }^{\mathrm{a}}$ \\ a INRAE, Abeilles et Environnement, 84914 Avignon, France \\ ${ }^{\mathrm{b}}$ Institute of Bee Health, University of Bern, 3003 Bern, Switzerland \\ ' Department of Entomology and Plant Pathology, North Carolina State University, Raleigh, NC, USA \\ ${ }^{\mathrm{d}}$ Department of Ecology, Swedish University of Agricultural Sciences, 75007 Uppsala, Sweden \\ e USDA, ARS Honey Bee Breeding, Genetics and Physiology Laboratory, Baton Rouge, LA, USA
}

\section{A R T I C L E I N F O}

Article history:

Received 4 December 2019

Received in revised form 12 February 2020

Accepted 24 March 2020

Available online 5 May 2020

\section{Keywords:}

Host resistance

Tolerance

Honey bee

Varroa destructor

Marker-assisted selection

Host-parasite interactions

\begin{abstract}
A B S T R A C T
The ectoparasitic mite Varroa destructor is the most significant pathological threat to the western honey bee, Apis mellifera, leading to the death of most colonies if left untreated. An alternative approach to chemical treatments is to selectively enhance heritable honey bee traits of resistance or tolerance to the mite through breeding programs, or select for naturally surviving untreated colonies. We conducted a literature review of all studies documenting traits of $A$. mellifera populations either selectively bred or naturally selected for resistance and tolerance to mite parasitism. This allowed us to conduct an analysis of the diversity, distribution and importance of the traits in different honey bee populations that can survive $V$. destructor globally. In a second analysis, we investigated the genetic bases of these different phenotypes by comparing 'omics studies (genomics, transcriptomics, and proteomics) of A. mellifera resistance and tolerance to the parasite. Altogether, this review provides a detailed overview of the current state of the research projects and breeding efforts against the most devastating parasite of $A$. mellifera. By highlighting the most promising traits of Varroa-surviving bees and our current knowledge on their genetic bases, this work will help direct future research efforts and selection programs to control this pest. Additionally, by comparing the diverse populations of honey bees that exhibit those traits, this review highlights the consequences of anthropogenic and natural selection in the interactions between hosts and parasites.

(C) 2020 The Authors. Published by Elsevier Ltd on behalf of Australian Society for Parasitology. This is an open access article under the CC BY-NC-ND license (http://creativecommons.org/licenses/by-nc-nd/4.0/).
\end{abstract}

\section{Introduction: towards a stable host-parasite relationship between Apis mellifera and varroa destructor?}

The ectoparasitic mite Varroa destructor (hereon Varroa) is indisputably the most significant pathological threat to the western honey bee, Apis mellifera, worldwide (Van Engelsdorp et al., 2008; Dietemann et al., 2012). In the wake of its global spread during the 1950-1990s, this parasite has severely impacted the management and profitability of beekeeping (Le Conte et al., 2010; Carreck and Neumann, 2010). The mite is completely dependent on the honey bee colony, with a reproductive cycle synchronized to host pupa development inside brood cells while feeding on brood and adult bee haemolymph and fat body tissue (Ramsey

\footnotetext{
* Corresponding author.

E-mail address: fanny.mondet@inra.fr (F. Mondet).

1 These authors contributed equally.
}

et al., 2019). However, the most devastating impact of the mite is that it is a biological vector for honey bee viruses (Martin et al., 2012; Mondet et al., 2014; Wilfert et al., 2016). In the absence of the mite, these viruses persist in colonies as covert infections. However, the opportunistic nature of these viruses, together with exponential mite population growth, quickly results in the development of lethal virus epidemics that typically kill a colony within 2-3 years (Amdam et al., 2004).

Colonies of the mite's natural host, the eastern honey bee (Apis cerana), are generally not threatened by $V$. destructor due to a stable host-parasite relationship that has been established over a long evolutionary scale (Oldroyd, 1999). Such a relationship is distinguishably missing with the new host, A. mellifera, which acquired the mite after colonies were transported into northeastern Asia (Kulikov, 1965; Crane, 1968). Apis cerana has a variety of defence mechanisms that limit the mite's population growth (Peng et al., 1987; Boecking et al., 1993; Page et al., 2016; 
Lin et al., 2018). While A. mellifera shares some of these defence mechanisms, they are far less pronounced (Fries et al., 1996). As a result, varroa mites are able to maximize reproductive opportunities, which ultimately results in exponential mite population growth to lethal levels (Martin et al., 1998; Calis et al., 1999).

Initial responses of researchers and beekeepers to varroa focused heavily on chemical treatments to control the mite. Today, most managed $A$. mellifera colonies depend on mite control treatments to survive (Rosenkranz et al., 2010). These chemical treatments can actually harm honey bees (Johnson et al., 2009; Locke et al., 2012a), leave residues in hive products (Johnson et al., 2013) and can become ineffective as $V$. destructor populations can swiftly become resistant (Milani, 1999; Beaurepaire et al., 2017). Additionally, mite control treatments also remove the selective pressure of natural mite infestation, preventing coevolutionary processes towards a stable host-parasite relationship (Neumann and Blacquière, 2016).

An alternative approach to reduce the dependency on chemical treatments has been to selectively enhance heritable resistance or tolerance to the mite through breeding programs (Büchler et al., 2010; Rinderer et al., 2010; see Fig. 1A for a timeline of breeding programs). This approach has yielded some success but it is tedious work and often based on genetically complex behaviour that is difficult to phenotype (Dekkers and Hospital, 2002; Beaurepaire et al., 2019a). However, recent advances in biotechnology can help facilitate selection. For instance, causative genes and proteins associated with resistance or tolerance can be developed as markerassisted selection (MAS) tools for improving breeding stock at a large scale (Grozinger and Robinson, 2015; Guarna et al., 2017).

In addition to selective breeding, natural selection has yielded honey bee populations in Europe, North America, South America and Africa that survive varroa without parasite management (Locke, 2016; Fig. 1A). The underlying mechanisms are not all well understood, and seem to vary between different naturally selected populations despite experiencing similar selection pressures (Locke et al., 2012b; Oddie et al., 2018a).
In this review, we conducted a literature survey of all studies documenting phenotypic features (i.e., traits) of $A$. mellifera populations either selectively bred or naturally selected for resistance and tolerance towards varroa. We evaluated 153 studies in total, published between 1984 and 2019 (Fig. 1B). For each study we reviewed, we asked the following questions: (i) Were the bees selectively bred or naturally selected? (ii) What are the investigated traits and are they similar across studies and populations? (iii) Are there common molecular pathways involved in different studies and populations? Our aim was to systematically evaluate the most promising traits of varroa-surviving bees to help direct future research efforts and selective breeding programs.

\section{Literature review of studies documenting resistance / tolerance / survival to varroa}

\subsection{History of research on honey bee resistance and tolerance traits}

Since $V$. destructor has only shared a short co-evolutionary history with $A$. mellifera, very little was known about the relationship between the novel host and the parasite. Investigations were therefore conducted on $A$. cerana, the original host, to decipher the mechanisms underlying the balanced host-parasite relationship in that bee species. Pioneering studies identified several mite resistance traits such as grooming behaviour (Peng et al., 1987), reduction of mite fertility and varroa-sensitive hygiene behaviour (VSH) (Rath and Drescher, 1990; Boot et al., 1999). Other passive characteristics such as a short post-capping duration for brood cells also contribute to host-parasite equilibrium in the native host. For example, the post-capping periods for worker and drone brood of $A$. cerana are approximately 11 and 14 days, respectively (Rosenkranz and Engels, 1994). Since a foundress (mother) mite lays female eggs once every $30 \mathrm{~h}$, and the daughter mite needs to be completely mature to survive when the host bee emerges (Martin, 1994), fewer daughter mites can be produced in an A. cerana brood compared with $A$. mellifera.
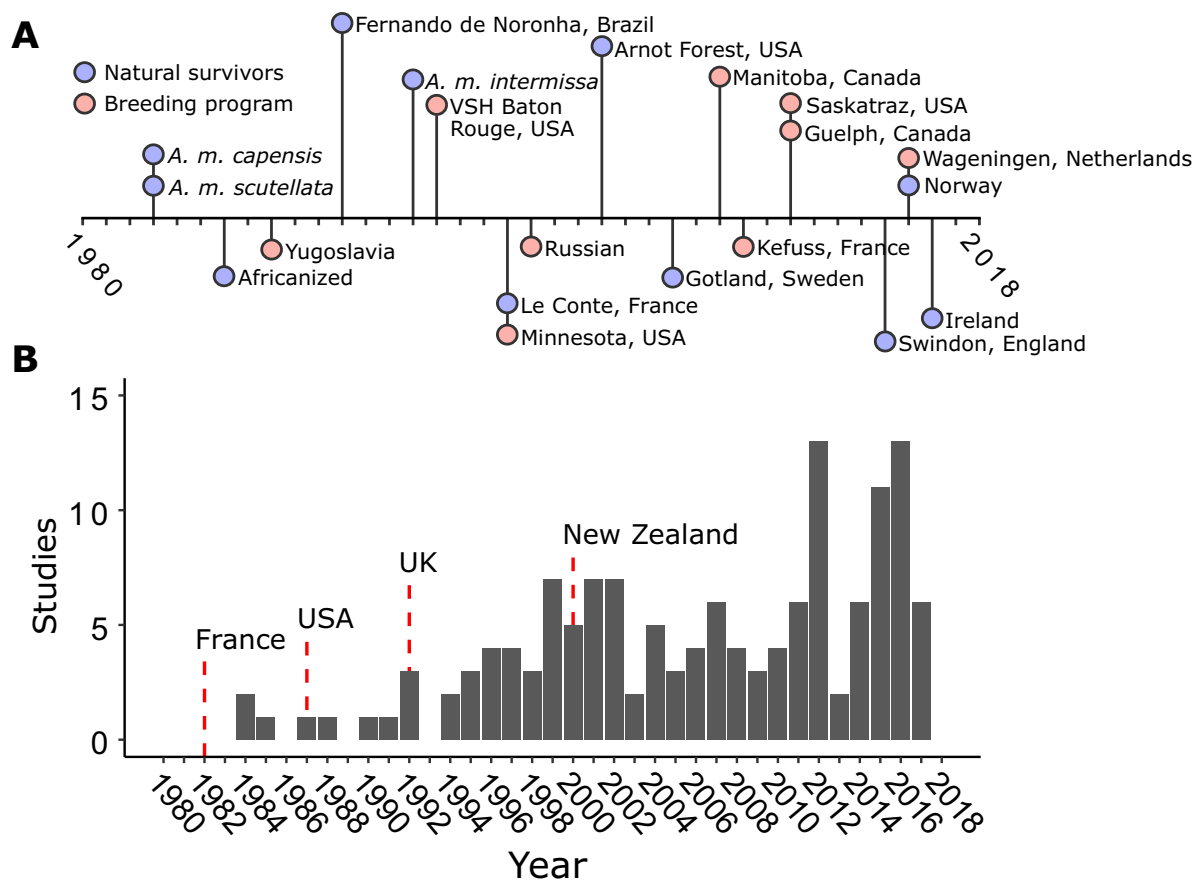

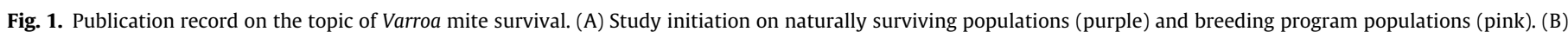

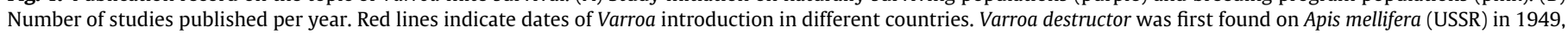
in Europe in 1967 (Bulgaria), in South America in 1971 (Paraguay) and in Africa in 1975 (Tunisia). 
In contrast to A. cerana, the development of A. mellifera worker brood typically takes 1 day longer; therefore, more female mites can reach maturity. However, two African Western honey bee subspecies - Apis mellifera capensis and Apis mellifera scutellata - have significantly shorter worker brood post-capping stages than Apis mellifera carnica (a European subspecies) (Moritz, 1985). These subspecies are better able to resist varroa mites (Moretto et al., 1995; Rosenkranz, 1999), providing a further link between postcapping duration and varroa resistance (Moritz, 1985).

Selectively breeding honey bees with improved varroa resistance would be beneficial for beekeeping and reduce dependence on acaricides. To this end, heritability analyses have been conducted to quantify the selection potential of traits that limit varroa population growth and efforts have been made to select resistant bees on this basis (Moritz, 1985; Le Conte et al., 1994; Harbo and Harris, 1999a; Boecking et al., 2000; Stanimirovic et al., 2008, 2010).

Another selection approach has been to leave colonies untreated, and to select the survivors for breeding. This risky approach, also called the "Bond Test", has been successfully developed in France (by J. Kefuss, France) and in Sweden (by I. Fries, SLU, Sweden). In some cases, no human interference was necessary, and colonies developed natural resistance or tolerance to the mite without human intervention. For example, two small populations in western and southeastern France exist as feral colonies which repopulated the area after they had been destroyed when varroa mites first invaded (Le Conte et al., 2007). Similar colonies have also been identified in the Arnot Forest in the northeastern USA (Seeley, 2007). However, the largest honey bee population that survives naturally with the mite are the Africanized bees in South America, Central America, and the southern United States (Rosenkranz, 1999). Naturally varroa-surviving populations may not be suitable for large-scale commercial beekeeping owing to undesirable beekeeping characteristics such as frequent swarming or low productivity, but they are precious for genetic diversity and biodiversity, as they can contribute to pollination in wild areas where managed honey bee colonies are less prevalent. Moreover, these colonies are good models for studying the mechanisms of a stable host-parasite relationship and thus to characterize the specific phenotypes that could be selected to support beekeeping.

\subsection{VSH: a case study to illustrate the temporal changes in our understanding of mechanisms for survival}

Behavioural traits are an important part of honey bees' social immunity repertoire (Cremer et al., 2007). However, these traits are complex, ranging from performances of single bees to groups of individuals performing different stages of what is considered a single activity (Spötter et al., 2016), making it difficult to do comparative research on expression of the behaviour and effective selection in breeding programs (Fonio et al., 2012; Bergman and Beehner, 2015).

A case study of research directed at this problem comes from a U.S. Department of Agriculture (USDA) laboratory in Louisiana, USA. In 1995, researchers identified colonies with negative mite population growth (MPG; Table 1) during a short (10 week) test under highly controlled experimental conditions (Harbo and Hoopingarner, 1997). The factor most strongly associated with low MPG was a high frequency of non-reproducing varroa foundresses. This characteristic had been observed previously in mite-resistant populations of $A$. mellifera in Uruguay (Ruttner et al., 1984), Tunisia (Ritter, 1990), and Argentina (Eguaras et al., 1995). The trait associated with high non-reproduction in the $U$. $\mathrm{S}$. bees came to be called suppressed mite reproduction (SMR) (Harbo and Harris, 1999a). SMR was determined to be heritable (Harbo and Harris, 1999b) and became the focus of selective breed-
Table 1

Definitions of the terminology related to honey bee survival with Varroa infestations.

\begin{tabular}{|c|c|}
\hline Terms & Definitions \\
\hline Host tolerance & $\begin{array}{l}\text { Ability of a host (honey bee colony or individual } \\
\text { bee) to reduce the impairment caused by the } \\
\text { parasite }\end{array}$ \\
\hline Host resistance & $\begin{array}{l}\text { Ability of a host (honey bee colony or individual } \\
\text { bee) to reduce the reproductive success of the } \\
\text { parasite so that the infestation stays below a } \\
\text { damaging level }\end{array}$ \\
\hline Honey bee trait & $\begin{array}{l}\text { An observable structural or functional feature of } \\
\text { honey bees or honey bee colonies }\end{array}$ \\
\hline Regulatory trait & $\begin{array}{l}\text { A heritable structural, physiological or behavioural } \\
\text { trait that confers resistance or tolerance to the host } \\
\text { against a parasite }\end{array}$ \\
\hline Mite fertility & $\begin{array}{l}\text { Natural ability of a foundress mite to produce at } \\
\text { least one egg }\end{array}$ \\
\hline Mite fecundity & $\begin{array}{l}\text { Abundance of eggs laid by a given foundress, or the } \\
\text { reproductive rate measured by the number of eggs } \\
\text { produced }\end{array}$ \\
\hline $\begin{array}{l}\text { Mite population } \\
\text { growth }\end{array}$ & $\begin{array}{l}\text { The change in the number of mites in a population } \\
\text { over a specified time. No (or low) population } \\
\text { growth is the basis of host resistance }\end{array}$ \\
\hline $\begin{array}{l}\text { Mite non-reproduction } \\
\quad(\text { MNR) }\end{array}$ & $\begin{array}{l}\text { Failure of a foundress mite to produce at least one } \\
\text { adult, mated female that will enter the colony's } \\
\text { mite population when the developing bee emerges } \\
\text { from the cell as an adult bee. A foundress mite will } \\
\text { not be successful at reproduction if she does not lay } \\
\text { any eggs (infertile), lays only one egg, produces no } \\
\text { male offspring or begins laying her eggs too late in } \\
\text { relation to the pupal development }\end{array}$ \\
\hline $\begin{array}{l}\text { Suppressed mite } \\
\quad \text { reproduction (SMR) }\end{array}$ & $\begin{array}{l}\text { Redefined as only cases of mite non-reproduction } \\
\text { that are regulated by traits expressed by the brood }\end{array}$ \\
\hline $\begin{array}{l}\text { Hygienic behaviour } \\
\text { (HYG) }\end{array}$ & $\begin{array}{l}\text { Behavioural sequence consisting of the targeting, } \\
\text { opening and removal of diseased, injured, } \\
\text { parasitized, or dead brood by worker bees. This trait } \\
\text { is usually assessed using the freeze-killed brood } \\
\text { (FKB) or pin-killed assays }\end{array}$ \\
\hline $\begin{array}{l}\text { Varroa-sensitive } \\
\text { hygiene (VSH) }\end{array}$ & $\begin{array}{l}\text { Form of hygienic behaviour that specifically targets } \\
\text { and removes brood infested by Varroa mites. This } \\
\text { trait is assessed through assays that measure the } \\
\text { removal of Varroa-parasitized brood }\end{array}$ \\
\hline Recapping & $\begin{array}{l}\text { Behavioural sequence consisting of the targeting, } \\
\text { opening and then recapping of brood cells, leading } \\
\text { to the potential disruption of mite reproduction. } \\
\text { More research is needed to confirm that this trait is } \\
\text { totally distinct from VSH }\end{array}$ \\
\hline Grooming & $\begin{array}{l}\text { Behaviour consisting of the removal of Varroa from } \\
\text { adult bees, either by a bee infested by a mite itself } \\
\text { (autogrooming) or by a bee cleaning another bee } \\
\text { (allogrooming) }\end{array}$ \\
\hline Mite virulence & $\begin{array}{l}\text { Ability of the parasite (mite) to inflict harm on its } \\
\text { host }\end{array}$ \\
\hline
\end{tabular}

ing for resistance (Harbo and Harris, 2003). While it is reasonable to expect that the brood of SMR bees somehow caused the poor mite reproduction (Milani et al., 2004), it was later discovered that the selected bees expressed high levels of the trait of hygiene (HYG). Thus, an effect of the adult bees, not the brood, was the major driver of high non-reproduction in mites (Harbo and Harris, 2005). Surprisingly, SMR bees removed more miteinfested pupae than bees which had been selectively bred for hygienic removal of freeze-killed brood (Spivak, 1996; Ibrahim and Spivak, 2006). Moreover, the hygienic activity of SMR bees appeared to be biased toward pupae parasitized by reproducing mites rather than non-reproducing mites (Harbo and Harris, 2005; Ibrahim and Spivak, 2006). This largely explained the higher proportion of non-reproducing mites in the SMR colonies, relative to unselected colonies. The SMR trait was thus renamed varroa sensitive hygiene (VSH) to emphasize the regulatory behavioural mechanism that governs high mite resistance (Harris, 2007).

Despite changes in understanding about the main mechanisms of mite resistance, selection for VSH has almost always been based 
on the frequency of non-reproducing mites in a colony (although there was some selection by quantifying removal of miteinfested brood). Traits other than HYG (e.g., disruptive semiochemical production by the brood or physiological modifications of the parasitized pupae) could contribute to high mite nonreproduction, and such mechanisms should be retained during selection. Indeed, compounds released by infested brood can compromise varroa reproduction and result in increased MNR, independently from an action of the adult bees (Nazzi and Milani, 1996, Milani et al., 2004). Recent evidence also suggests that there may be a brood effect contributing to HYG and VSH (Wagoner et al., 2018, 2019) although such an effect has been difficult to stabilize through breeding (Villa et al., 2016). We propose here to rename the phenotypic feature of high mite non-reproduction as MNR because it may be derived from both VSH activity and an effect of bee brood (Table 1). We recommend reserving the term SMR for non-reproduction of mites that is induced solely by the brood.

\subsection{Qualitative and quantitative analyses of traits related to A. mellifera survival with Varroa}

We conducted a literature survey to understand the traits linked to the ability of honey bees to survive varroa after natural selection or targeted breeding. Publications were identified through a comprehensive search on Web of Science (last updated in March 2019), using the search string: 'varroa AND [surv* OR resist* OR tol*]'. A filter was applied to include only studies performed on colonies that had been untreated for varroa for a minimum of 2 years, to meet the definition of 'surviving' (Mondet and Conte, 2014). We identified 78 studies, corresponding to 20 different surviving populations worldwide (Table 2, Fig. 1A).

Among the different $A$. mellifera populations, Africanized honey bees are the most studied, with 19 publications focusing on this group, mainly in Brazil and Mexico (Fig. 2). The second and third most studied populations are honey bees specifically bred by the USDA for the SMR/MNR and VSH traits (VSH Baton Rouge), or for low MPG (Russian bees from Primorsky), with 10 and eight publications, respectively. The remaining populations have been reported in one to six publications each. This highlights the heterogeneity of the different populations which have been investigated. Individual studies were performed on between one and 100 colonies each, with a mean of 12 colonies per study and population $(+/-10.6)$, leaving doubts on the importance of some traits in specific populations (Table 2). The observations were made between 1983 and 2017, showing that the mechanistic study of honey bee survival with Varroa infestations has an historic precedence, which started shortly after the introduction of the mites in western Europe (Ruttner and Ritter, 1980) (Fig. 1B).

Many phenotypes have been studied in the surviving populations of $A$. mellifera, ranging from individual bee to colony phenotypes (Tables 2 and 3). Varroa population dynamics resulting in reduced MPG, as measured by brood infestation, phoretic infestation, or natural mite mortality, has been investigated in approximately $40 \%$ of the studies ( 31 publications). Low MPG has been confirmed in 25 out of these 31 studies, comprising $95 \%$ of the studied populations (19 out of 20). This confirms that in most surviving populations, the ability of colonies to maintain mite numbers below a damaging level is a central feature. Varroa resistance mechanisms, rather than tolerance mechanisms, are thus more likely to explain honey bee survival with Varroa infestations.

We distinguished 15 putative mechanisms that were investigated and further confirmed as primary regulatory traits that are involved in the survival of honey bee colonies with Varroa infestations (Table 3). The literature review approach for comparative phenotypic analyses should be interpreted with care since several factors can influence publication frequency beyond the importance of a given trait in a population. When considering all $A$. mellifera populations, grooming appears as the most studied trait (18 studies), followed by VSH and MNR (17 studies each) (Fig. 3). MNR was the trait confirmed in most studies (14 studies, seven populations), followed by VSH (10 studies, five populations) and grooming (10 studies, five populations).

Interestingly, beyond MNR and VSH, recapping is also frequently verified to have an important role; it has been identified in seven different studies documenting eight different populations (Fig. 3). By recapping cells, it is believed that honey bees can disrupt the mite's reproductive cycle and cause lower fecundity of the foundress (Oddie et al., 2019). Although researchers began investigating this trait in the mid-1990s (Aumeier et al., 2000), we notice a re-emerging interest in recapping in the recent years. The relative contribution of this trait to varroa resistance is still debated and needs further investigation (Van Alphen and Fernhout, 2019).

There has been some disagreement in the literature over the contributing role of HYG, as selected using the freeze-killed brood or pin-test assays (Leclercq et al., 2018), for varroa resistance. HYG has been extensively tested in surviving populations, with eight studies investigating it and one confirming its potential involvement in honey bee survival with Varroa infestations (Table 2). Confusion exists around the concepts of VSH and HYG, and their respective associated assays. The behavioural motor sequence is identical in both cases: upon detection of a stimulus, workers uncap and remove the targeted cell contents. What remains unclear, however, is whether the detection step occurs through identical stimuli. Evidence suggests that it may not be the case (Nazzi et al., 2004; Mondet et al., 2016; McAfee et al., 2018; Wagoner et al., 2019). VSH is unarguably a specific form of HYG, but the freeze-killed brood or pin-test methods used to score HYG often fail to identify colonies that display VSH. Selectively bred VSH colonies (VSH BR population) adeptly remove freezekilled brood, but bees bred for HYG using the freeze-killed brood assay (MIN population) remove low proportions of varroainfested brood (Danka et al., 2013). Some studies confirmed that colonies bred for HYG displayed lower mite levels than control colonies (Spivak, 1996; Spivak and Reuter 1998; Ibrahim and Spivak, 2006, 2007), but since these studies were not performed on colonies untreated for at least 2 years, they were not included in the literature review. The sole use of the freeze-killed brood or pin-test assays does not appear to be sufficient to select for varroa resistance. Other assays that more directly quantify the removal of mite-infested brood are better tools to select for varroa resistance.

Excluding Africanized bees, A. m. scutellata, A. m. capensis and $A$. $m$. intermissa from the literature review, we found that the most frequently confirmed traits are MNR (11 studies, five populations), VSH (eight studies, four populations) and recapping (seven studies, six populations), with fewer studies confirming grooming (three studies and three populations, Table 3).

These comparative phenotypic analyses highlight the diversity of traits that appear to play roles in different surviving populations; no universal mechanism for survival emerged from this literature survey. In most cases, survival of both naturally and artificially selected populations is due to the expression of several traits that appear to collectively confer resilience to varroa infestation (Fig. 3). This seems true for the naturally selected populations as well as for the populations specifically bred for one or several traits. For the three best-documented naturally selected populations (Arnot Forest - USA, Gotland - Sweden, Le Conte - France), four to six traits seem to enhance varroa-survival abilities of bee colonies, highlighting the complexity of maintaining a stable host-parasite equilibrium. Interestingly, in populations selected 
Table 2

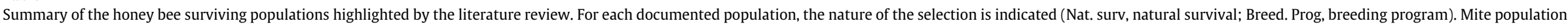
growth (MPG, indicated in italics) represents a downstream result of traits.

\begin{tabular}{|c|c|c|c|c|c|c|c|c|c|c|c|c|}
\hline Population & Country & $\begin{array}{l}\text { No. } \\
\text { studies }\end{array}$ & Selection & $\begin{array}{l}\text { Selection } \\
\text { criteria }\end{array}$ & $\begin{array}{l}\text { Years } \\
\text { no } \\
\text { treat }\end{array}$ & $\begin{array}{l}\text { No. } \\
\text { investigated } \\
\text { traits }\end{array}$ & Investigated traits & $\begin{array}{l}\text { No. } \\
\text { verified } \\
\text { traits }\end{array}$ & Verified traits & $\begin{array}{l}\text { No. } \\
\text { colonies }\end{array}$ & $\begin{array}{l}\text { Study } \\
\text { years }\end{array}$ & Refs \\
\hline Africanized & $\begin{array}{l}\text { Brazil, } \\
\text { Mexico } \\
\text { Puerto Rico }\end{array}$ & 19 & Nat. surv. & - & $\begin{array}{l}\text { No } \\
\text { treat }\end{array}$ & 13 & $\begin{array}{l}\text { Grooming, VSH, recapping, } \\
\text { brood susceptibility, mite } \\
\text { fertility, cell size, HYG, mite } \\
\text { fecundity, aggressiveness, } \\
\text { MNR, mite haplotype, brood } \\
\text { attractiveness, MPG }\end{array}$ & 8 & $\begin{array}{l}\text { Grooming, } \\
\text { VSH, mite } \\
\text { infertility, cell } \\
\text { size, MNR, } \\
\text { mite } \\
\text { haplotype, } \\
\text { mite } \\
\text { fecundity, } \\
\text { MPG }\end{array}$ & $3-29$ & $\begin{array}{l}1986- \\
2010\end{array}$ & $\begin{array}{l}\text { Rosenkranz and Engels (1994), Message and } \\
\text { Goncalves (1995), Aumeier et al. (1996, 2000, } \\
\text { 2002), Corrêa-Marques and De Jong (1998), } \\
\text { Guzman-Novoa et al. (1999, 2012), Medina } \\
\text { and Martin (1999), Guerra (2000), Aumeier } \\
\text { (2001), Vandame et al. (2002), Garrido et al. } \\
\text { (2003), Mondragon et al. (2005, 2006), } \\
\text { Carneiro et al. (2007), Strapazzon et al. } \\
\text { (2009), Pinto et al. (2012), Rivera-Marchand } \\
\text { et al. (2012) }\end{array}$ \\
\hline Arnot Forest & USA & 5 & Nat. surv. & - & $\begin{array}{l}\text { No } \\
\text { treat }\end{array}$ & 5 & $\begin{array}{l}\text { Small colonies, swarming, } \\
\text { genetic isolation, high queen } \\
\text { polyandry, } M P G\end{array}$ & 3 & $\begin{array}{l}\text { Small hives, } \\
\text { swarming, } \\
\text { genetic } \\
\text { isolation, MPG }\end{array}$ & $6-23$ & $\begin{array}{l}2002- \\
2016\end{array}$ & $\begin{array}{l}\text { Seeley (2007, 2017), Seeley et al. (2015), } \\
\text { Tarpy et al. (2015), Loftus et al. (2016) }\end{array}$ \\
\hline Capensis & South Af. & 2 & Nat. surv. & - & $\begin{array}{l}\text { No } \\
\text { treat }\end{array}$ & 2 & Post capping duration, $M P G$ & 2 & $\begin{array}{l}\text { Short post } \\
\text { capping stage, } \\
M P G\end{array}$ & 5 & $\begin{array}{l}1983- \\
1988\end{array}$ & Moritz et al. $(1984,1990)$ \\
\hline $\begin{array}{l}\text { Fernando de } \\
\text { Noronha }\end{array}$ & Brazil & 2 & Nat. surv. & - & $2-15$ & 3 & Grooming, mite fertility, MPG & 1 & MPG & $12-20$ & $\begin{array}{l}1991- \\
1997\end{array}$ & $\begin{array}{l}\text { de Jong and Soares (1997), Corrêa-Marques } \\
\text { et al. (2002) }\end{array}$ \\
\hline Gotland & Sweden & 6 & Nat. surv. & - & $6-20$ & 8 & $\begin{array}{l}\text { Swarming, grooming, HYG, } \\
\text { colony size, MNR, virus } \\
\text { tolerance and resistance, } \\
\text { recapping, } M P G\end{array}$ & 6 & $\begin{array}{l}\text { Colony size, } \\
\text { MNR, virus } \\
\text { tolerance and } \\
\text { resistance, } \\
\text { recapping, } \\
\text { MPG }\end{array}$ & $4-23$ & $\begin{array}{l}2005- \\
2016\end{array}$ & $\begin{array}{l}\text { Fries et al. (2006), Fries and Bommarco } \\
\text { (2007), Locke et al. (2012a,b, 2014), Oddie } \\
\text { et al. (2018a,b) }\end{array}$ \\
\hline Guelph & Canada & 1 & $\begin{array}{l}\text { Breed. } \\
\text { prog. }\end{array}$ & MPG & 1 & 2 & Grooming, $M P G$ & 1 & $\begin{array}{l}\text { Grooming, } \\
\text { MPG }\end{array}$ & 13 & 2010 & Guzmán-Novoa et al. (2012) \\
\hline Intermissa & Algeria & 1 & Nat. surv. & - & $2-9$ & 1 & $M P G$ & 1 & MPG & 9 & $\begin{array}{l}1994- \\
2002\end{array}$ & Kefuss et al. (2004) \\
\hline Ireland & Ireland & 1 & Nat. surv. & - & 6 & 3 & $\begin{array}{l}\text { Grooming, DWV tolerance/ } \\
\text { resistance, MPG }\end{array}$ & 1 & $M P G$ & 5 & 2016 & McMullan (2018) \\
\hline Kefuss & France & 1 & $\begin{array}{l}\text { Breed. } \\
\text { prog. }\end{array}$ & Survival & 11 & 2 & HYG, MPG & 1 & MPG & $\sim 100$ & 2008 & Kefuss et al. (2015) \\
\hline Le Conte & France & 6 & Nat. surv. & - & $2-13$ & 7 & $\begin{array}{l}\text { Swarming, MNR, grooming, } \\
\text { VSH, recapping, propolis, MPG }\end{array}$ & 6 & $\begin{array}{l}\text { Swarming, } \\
\text { MNR, VSH, } \\
\text { recapping, } \\
\text { propolis, MPG }\end{array}$ & $2-21$ & $\begin{array}{l}1998- \\
2016\end{array}$ & $\begin{array}{l}\text { Martin et al. (2001), Le Conte et al. (2007), } \\
\text { Navajas et al. (2008), Locke et al. (2012), } \\
\text { Popova et al. (2014), Oddie et al. (2018a,b) }\end{array}$ \\
\hline Manitoba & Canada & 1 & $\begin{array}{l}\text { Breed. } \\
\text { prog. }\end{array}$ & $\begin{array}{l}\text { Survival/ } \\
\text { MPG }\end{array}$ & 12 & 2 & Grooming, MPG & 2 & $\begin{array}{l}\text { Grooming, } \\
M P G\end{array}$ & 12 & 2007 & Bahreini and Currie (2015) \\
\hline $\begin{array}{l}\text { Minnesota } \\
\text { (MIN) }\end{array}$ & USA & 3 & $\begin{array}{l}\text { Breed. } \\
\text { prog. }\end{array}$ & HYG & $2-12$ & 4 & VSH, MNR, recapping, MPG & 3 & $\begin{array}{l}\text { VSH, } \\
\text { recapping, } \\
\text { MPG }\end{array}$ & $3-63$ & $\begin{array}{l}1998- \\
2011\end{array}$ & $\begin{array}{l}\text { Spivak and Reuter (2001), Ibrahim and Spivak } \\
\text { (2006), Ibrahim et al. (2007) }\end{array}$ \\
\hline Norway & Norway & 3 & Nat. surv. & - & $17-19$ & 6 & $\begin{array}{l}\text { MNR, grooming, VSH, } \\
\text { recapping, post-capping } \\
\text { duration, MPG }\end{array}$ & 4 & $\begin{array}{l}\text { MNR, } \\
\text { recapping, } \\
\text { post-capping } \\
\text { duration, MPG }\end{array}$ & 5 & $\begin{array}{l}2015- \\
2017\end{array}$ & Oddie et al. (2017, 2018a,b, 2019) \\
\hline Russian & USA & 8 & $\begin{array}{l}\text { Breed. } \\
\text { prog. }\end{array}$ & MPG & $11-15$ & 6 & $\begin{array}{l}\text { MNR, grooming, DWV } \\
\text { tolerance/resistance, VSH, } \\
\text { recapping, MPG }\end{array}$ & 6 & $\begin{array}{l}\text { MNR, } \\
\text { grooming, } \\
\text { DWV } \\
\text { tolerance/ } \\
\text { resistance, } \\
\text { VSH, }\end{array}$ & $4-32$ & $\begin{array}{l}1999- \\
2013\end{array}$ & $\begin{array}{l}\text { Rinderer et al. (1999, 2001), Harris and } \\
\text { Rinderer (2004), De Guzman et al. (2008), } \\
\text { Guzmán-Novoa et al. (2012), Kirrane et al. } \\
(2015,2018), \text { Khongphinitbunjong et al. } \\
(2016)\end{array}$ \\
\hline
\end{tabular}


Table 2 (continued)

\begin{tabular}{|c|c|c|c|c|c|c|c|c|c|c|c|c|}
\hline Population & Country & $\begin{array}{l}\text { No. } \\
\text { studies }\end{array}$ & Selection & $\begin{array}{l}\text { Selection } \\
\text { criteria }\end{array}$ & $\begin{array}{l}\text { Years } \\
\text { no } \\
\text { treat }\end{array}$ & $\begin{array}{l}\text { No. } \\
\text { investigated } \\
\text { traits }\end{array}$ & Investigated traits & $\begin{array}{l}\text { No. } \\
\text { verified } \\
\text { traits }\end{array}$ & Verified traits & $\begin{array}{l}\text { No. } \\
\text { colonies }\end{array}$ & $\begin{array}{l}\text { Study } \\
\text { years }\end{array}$ & Refs \\
\hline & & & & & & & & & $\begin{array}{l}\text { recapping, } \\
M P G\end{array}$ & & & \\
\hline Saskatraz & USA & 1 & Nat. surv. & - & 11 & 3 & $\begin{array}{l}\text { Brood susceptibility, pathogen } \\
\text { prevalence, } M P G\end{array}$ & 3 & $\begin{array}{l}\text { Brood } \\
\text { susceptibility, } \\
\text { pathogen } \\
\text { prevalence, } \\
\text { MPG }\end{array}$ & 1 & 2010 & Robertson et al. (2014) \\
\hline Scutellata & South Af. & 5 & Nat. surv. & - & $\begin{array}{l}\text { No } \\
\text { treat }\end{array}$ & 6 & $\begin{array}{l}\text { VSH, post-capping duration, } \\
\text { MNR, pathogen prevalence, } \\
\text { brood susceptibility, MPG }\end{array}$ & 4 & $\begin{array}{l}\text { Post-capping } \\
\text { duration, } \\
\text { MNR, } \\
\text { pathogen } \\
\text { prevalence, } \\
\text { MPG }\end{array}$ & $5-20$ & $\begin{array}{l}1983- \\
2018\end{array}$ & $\begin{array}{l}\text { Moritz (1985), Strauss et al. (2013, 2016), } \\
\text { Cheruiyot et al. (2018), Nganso et al. (2018) }\end{array}$ \\
\hline Swindon & UK & 1 & Nat. surv. & - & 18 & 1 & DWV tolerance & 1 & $\begin{array}{l}\text { DWV } \\
\text { tolerance }\end{array}$ & 3 & 2014 & Mordecai et al. (2016) \\
\hline VSH BR & USA & 11 & $\begin{array}{l}\text { Breed. } \\
\text { prog. }\end{array}$ & $\begin{array}{l}\text { SMR/VSH/ } \\
\text { MPG }\end{array}$ & $5-17$ & 8 & $\begin{array}{l}\text { HYG, VSH, MNR, recapping, } \\
\text { grooming, DWV tolerance/ } \\
\text { resistance, MPG }\end{array}$ & 6 & $\begin{array}{l}\text { HYG, VSH, } \\
\text { MNR, } \\
\text { recapping, } \\
\text { DWV } \\
\text { tolerance/ } \\
\text { resistance, } \\
\text { MPG }\end{array}$ & $1-43$ & $\begin{array}{l}1995- \\
2016\end{array}$ & $\begin{array}{l}\text { Harbo and Hoopingarner (1997), Harbo and } \\
\text { Harris (2005), Ibrahim et al. (2006, 2007), } \\
\text { Harris et al. (2010, 2012), Le Conte et al. } \\
\text { (2011), Tsuruda et al. (2012), Danka et al. } \\
(2013,2016)\end{array}$ \\
\hline Wageningen & Netherlands & 2 & $\begin{array}{l}\text { Breed. } \\
\text { prog. }\end{array}$ & Survival & $7-8$ & 3 & Grooming, VSH, MNR & 1 & VSH & 5 & $\begin{array}{l}2015- \\
2016\end{array}$ & $\begin{array}{l}\text { Kruitwagen et al. (2017), Panziera et al. } \\
\text { (2017) }\end{array}$ \\
\hline Yugoslavia & Yugoslavia & 1 & $\begin{array}{l}\text { Breed. } \\
\text { prog. }\end{array}$ & Survival & 4 & 1 & $M P G$ & 1 & MPG & 9 & 1988 & Kulinčević et al. (1992) \\
\hline
\end{tabular}

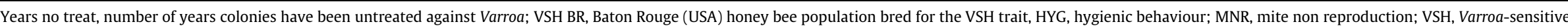
hygiene; DWV, deformed wing virus; Af, Africa. 


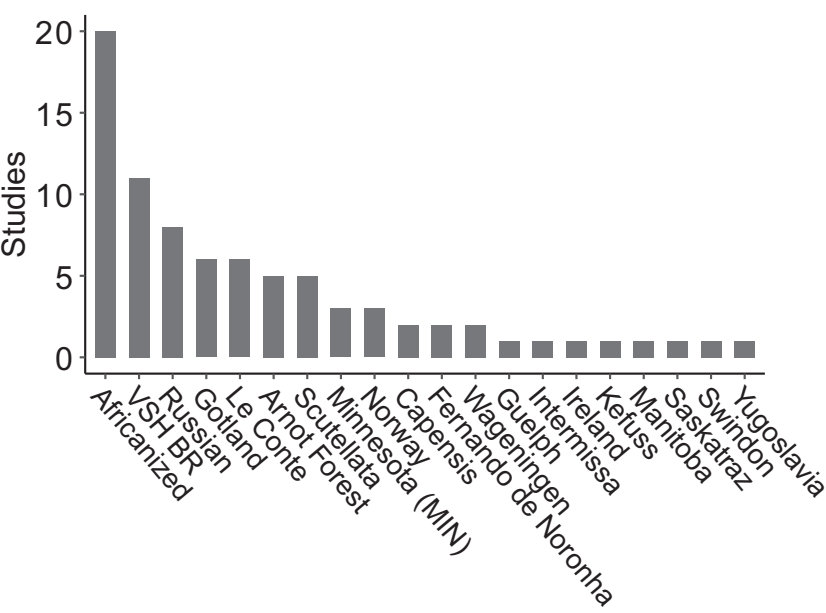

Fig. 2. Number of studies published for naturally Varroa-surviving populations and breeding program populations of honey bees. Africanized, Africanized honey bees (Brazil and Mexico); VSH BR, Baton Rouge VSH (Varroa-sensitive hygiene bred population (USA); Russian, Baton Rouge Russian bred population (USA); Gotland, Gotland Island surviving population (Sweden); Le Conte, Sarthe and Avignon surviving populations (France); Arnot Forest, Arnot Forest surviving populations (USA); Scutellata, Apis mellifera scutellata honey bees (South Africa); Minnesota, Minnesota hygienic bred population (USA); Norway, Norway surviving population; Capensis, Apis mellifera capensis honey bees (South Africa); F de Noronha, Fernando de Noronha surviving population (Brazil); Wageningen, Wageningen surviving populations (Netherlands); Guelph, Guelph breeding program (Canada); Intermissa, Apis mellifera intermissa surviving population (Algeria); Ireland, surviving population (Ireland); Kefuss, Kefuss surviving population (France); Manitoba, Manitoba breeding program (Canada); Saskatraz, Saskatraz surviving population (USA); Swindon, Swindon surviving population (UK); Yugoslavia, Yugoslavia breeding program (Yugoslavia).

Table 3

Summary of the regulatory traits governing honey bee survival with Varroa infestation, and associated proximate results.

\begin{tabular}{|c|c|c|}
\hline Mechanism & Regulatory trait & Proximate result(s) \\
\hline \multirow[t]{15}{*}{ Resistance } & Adult bees & \\
\hline & Grooming & Phoretic infestation $\downarrow$ \\
\hline & VSH & MNR $\uparrow$; Brood infestation $\downarrow$ \\
\hline & Recapping & MNR $\uparrow ;$ Fecundity $\downarrow$ \\
\hline & HYG & Brood infestation $\downarrow$ \\
\hline & Cell size & Fecundity $\downarrow$ \\
\hline & Brood & \\
\hline & $\begin{array}{l}\text { "SMR" (as a true brood-based } \\
\text { effect) }\end{array}$ & MNR $\uparrow$; Fecundity $\downarrow$ \\
\hline & Brood attractiveness & $\begin{array}{l}\text { Phoretic/brood infestation } \\
\text { ratio } \uparrow\end{array}$ \\
\hline & Post-capping duration & Fecundity $\downarrow$ \\
\hline & $\begin{array}{l}\text { High brood susceptibility } \\
\text { Colony }\end{array}$ & MNR $\uparrow$; Mortality $\uparrow$ \\
\hline & Swarming & Phoretic infestation $\downarrow$ \\
\hline & Small colonies & Brood infestation $\downarrow$ \\
\hline & Mites & \\
\hline & Mite haplotype & $\begin{array}{l}\text { MNR } \uparrow \text {; Fecundity } \downarrow \text {; } \\
\text { Mortality } \uparrow\end{array}$ \\
\hline \multirow[t]{3}{*}{ Tolerance } & $\begin{array}{l}\text { Pathogen tolerance (virus, } \\
\text { nosema, chalkbrood) }\end{array}$ & $\begin{array}{l}\text { Better colony survival with } \\
\text { high mite loads }\end{array}$ \\
\hline & Self-medication/Propolis & $\begin{array}{l}\text { Better colony survival with } \\
\text { high mite loads }\end{array}$ \\
\hline & Low brood susceptibility & $\begin{array}{l}\text { Better colony survival with } \\
\text { high mite loads }\end{array}$ \\
\hline
\end{tabular}

VSH, Varroa-sensitive hygiene; SMR, suppressed mite reproduction; MNR, mite non reproduction; HYG, hygienic behaviour.

and bred for specific phenotypes, several traits are also frequently found. The two best examples are the VSH Baton Rouge population (selected for low MPG, MNR and VSH) and the Russian population (selected for low MPG). Five and six traits were verified for each of

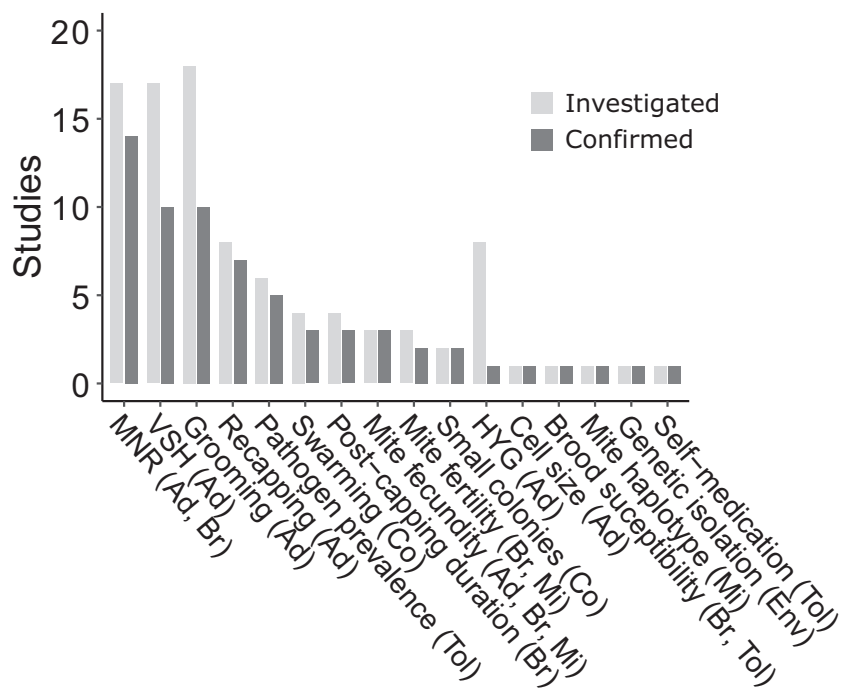

Fig. 3. Phenotypic features of honey bee populations that survive Varroa infestations. Number of studies investigating (light grey) or confirming (dark grey) each trait potentially involved in honey bee survival with Varroa infestations. Resistance traits can be attributed to an action from adult bees (Ad), the brood ( $\mathrm{Br}$ ), the colony (Co), mites (Mi) or environmental factors (Env). Tol, tolerance trait. MNR, Mite non reproduction; VSH, Varroa-sensitive hygiene, HYG, hygienic behaviour.

these two populations, respectively (Table 2). However, for the VSH Baton Rouge population, VSH is the primary trait that arose through selecting for high MNR, while the other traits are thought to contribute much less to mite resistance. Nevertheless, the repeated co-occurrence of traits in honey bee colonies with enhanced survival with Varroa infestations suggests that some of these traits might be related and regulated by common pathways and/or environmental parameters.

We urge researchers to distinguish the true regulatory mechanisms that are at the basis of bee survival with Varroa infestations (and which are at least partly genetically regulated) from the downstream results of those traits (Table 3, Fig. 4). Among the documented traits, five adult bee features (VSH, grooming, recapping, HYG, cell size), four brood features (MNR, post-capping duration, attractiveness, social apoptosis through high brood susceptibility), two colony features (swarming, colony size), one mite feature (virulence), and three tolerance features (tolerance of pathogens, selfmedication through propolis, low brood susceptibility) stand as regulatory traits. On the other hand, parameters such as mite population dynamics, MNR and MPG appear as downstream consequences. Environmental conditions (such as climate and nutrition) and beekeeping practises can also actively contribute to honey bee survival with Varroa infestations (Fig. 4). Some of these traits have been verified by only a few studies or in studies performed mainly on colonies which had been treated against varroa, and thus need confirmation.

\section{Review of studies using 'omics techniques to identify candidate genes for resistance, tolerance and survival}

\subsection{How can 'omics help us select for colony survival?}

'Omics tools have helped identify molecular markers to support selective breeding and to understand the molecular mechanisms underlying varroa resistance, tolerance and survival traits. Genomics is used to identify DNA fragments (single nucleotide polymorphisms (SNPs) or quantitative trait loci (QTLs)), transcriptomics quantifies RNA expression of candidate causal genes, and proteomics quantifies protein abundance. Theoretically, any of these 

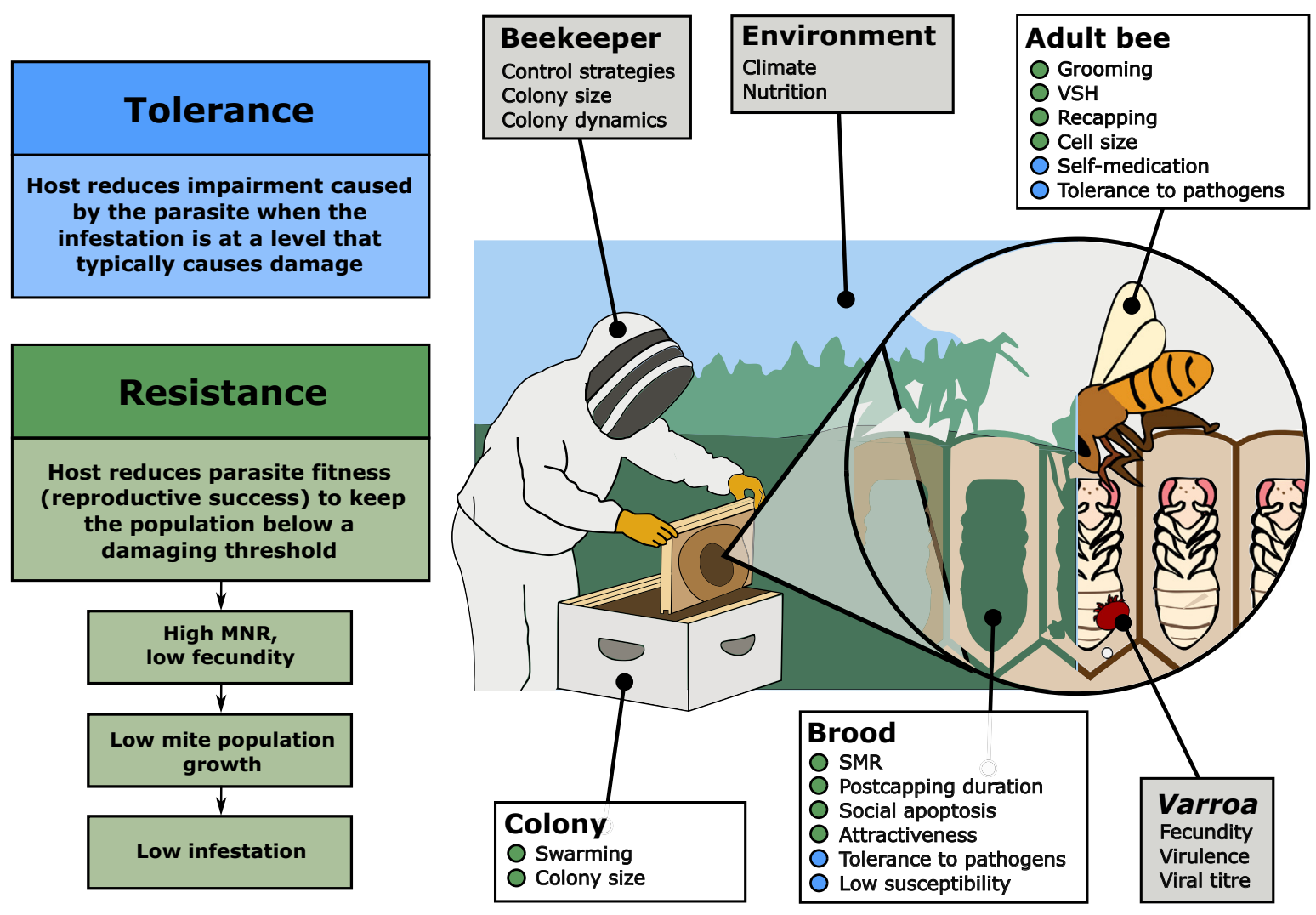

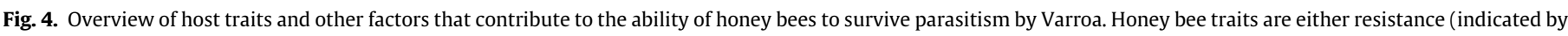

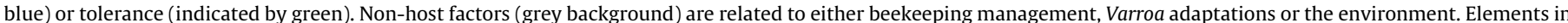

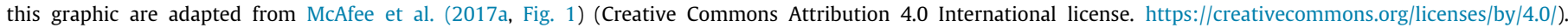
SMR, suppressed mite reproduction; VSH, Varroa sensitive hygiene.

three types of molecular markers could be used to select and improve the bee stocks, and the 'omics disciplines have greatly contributed to our understanding of the factors shaping honey bee health (Grozinger and Robinson, 2015; Trapp et al., 2017; Doublet et al., 2017). A multitude of studies investigating similar traits have now been conducted on honey bee populations surviving varroa infestation, which provides opportunities for new perspectives on specific molecular mechanisms and marker-assisted selection (MAS).

\subsection{Identifying social immunity pathways}

In order to understand whether resistance traits identified in populations of $A$. mellifera share the same genetic basis, we compared all relevant 'omics studies available on this topic. In all, 27 studies were included in the analysis (references in Supplementary Table S1). We also included analyses of HYG, which is not specific to varroa (see Section 2.3), to test whether the genetic basis of this trait and other varroa resistance traits are correlated. We first drew a map of the 16 A. mellifera chromosomes based on the size in Mbp from the Amel 4.5 assembly (Weinstock et al., 2006), which enabled us to include older studies. We then extracted locus information of the putative QTLs or SNPs involved in the resistance traits. Whenever locations were given in $\mathrm{cM}$, they were converted to bp based on recombination frequencies reported in Beye et al. (2006) (19 cM/Mb). Given the low genome-wide variation in recombination rate, this ratio was kept constant for all chromosomes. If available and extractable, information from transcriptomic studies was added to the scheme, excluding studies evaluating <25 genes (Supplementary Table S1). We converted Differentially Expressed Genes (DEGs) reported in these studies in old and new Beebase IDs (hymenopteragenome.org, metazoan.ensembl.org) whenever possible and obtained their location on the honey bee genome from the Ensembl Genome database (Kersey et al., 2018) and the Hymenoptera Genome Database (Elsik et al., 2018). This analysis resulted in 159 DEGs found in at least two of the eight studies, of which we could retrieve information on the location on the genome for 118 DEGs.

The most striking result from this literature survey is the lack of overlap in the findings across studies (Fig. 5). This has several potential explanations: (i) the different traits do not involve the same genetic pathways, (ii) the subspecies or populations of $A$. mellifera are different across studies and express the same trait through different molecular pathways, (iii) the protocols used to phenotype the bees are different and (iv) the technologies used are different. False positives (controlled between $1 \%$ and $10 \%$ depending on the study) may explain some of these differences, but not to the magnitude we observe here.

We expected that different varroa resistance traits may, in part, rely on the same sensory pathways (e.g. olfaction is likely involved in both VSH and HYG, and odorant binding protein 3 expression has been linked to grooming) (Guarna et al., 2017). Indeed, some loci appear to be linked to more than one social immunity trait (Fig. 5). For instance, some of the SNPs from the HYG investigations of Harpur et al. (2019) fall within the locus found by Tsuruda et al. (2012) when investigating VSH. However, more often, studies did not report the same regions to be involved, whether they were investigating the same trait or not. 


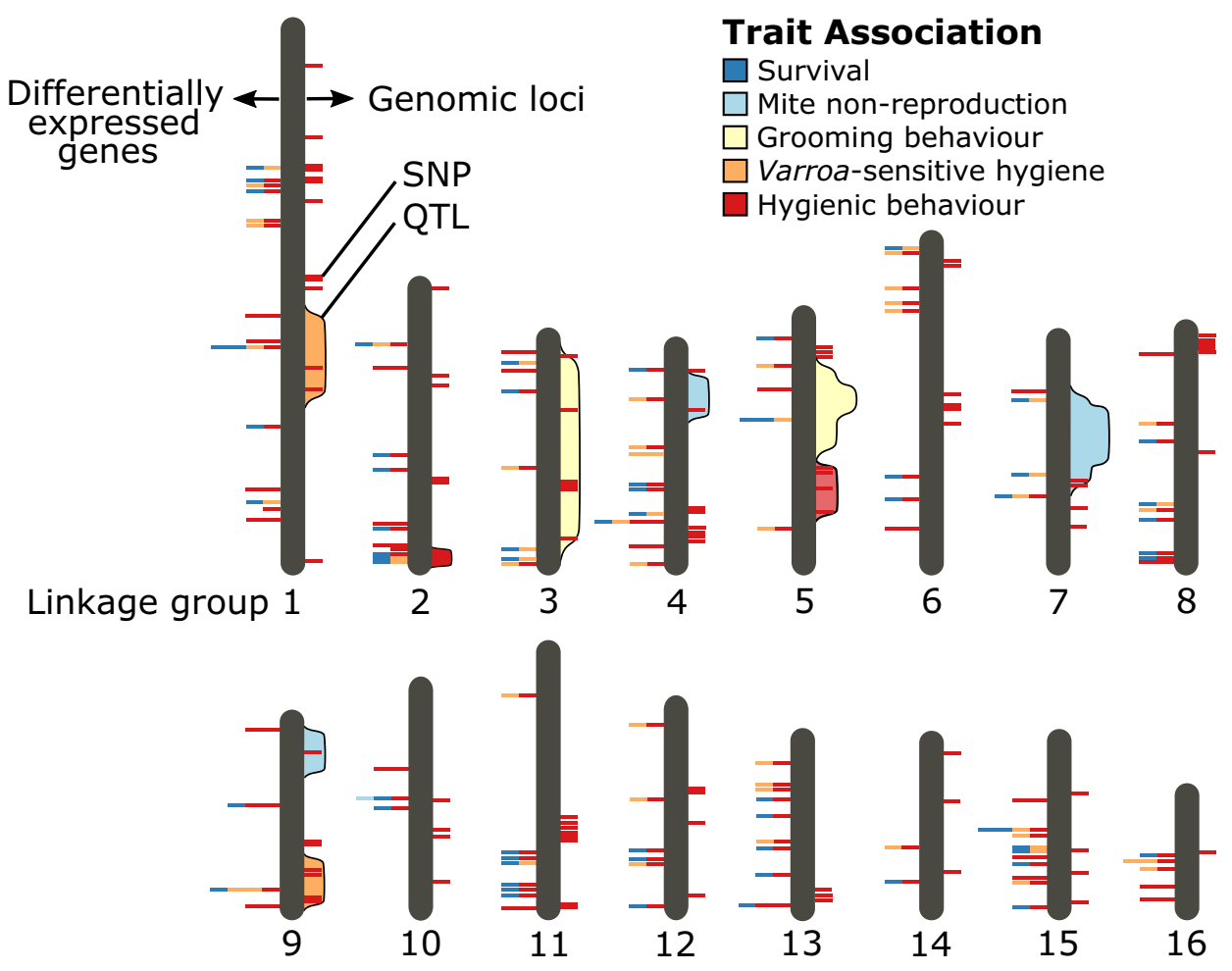

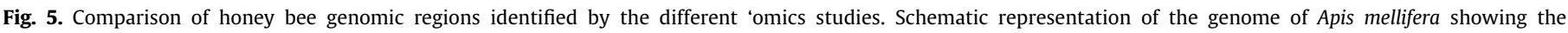

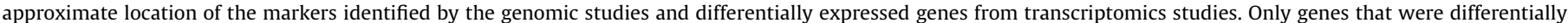

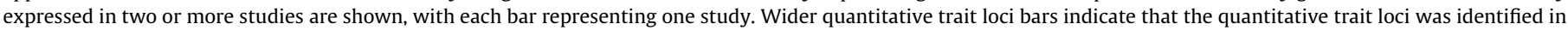

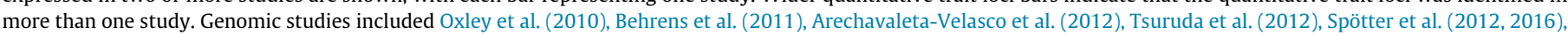

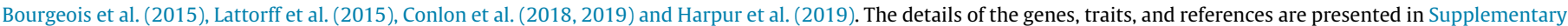
Tables S2-S4. SNP, single nucleotide polymorphism.

The diversity of $A$. mellifera populations with resistance or tolerance to $V$. destructor, together with the fact that these populations are located in very different geographic regions, suggest that mechanisms may have evolved independently in separate populations. Indeed, reports of convergent evolution for disease resistance in nature are rare (Meyers et al., 2005). Furthermore, duplicated genes, genes involved in the same physiological process, or different genes that achieve the same phenotypic outcome, may be blurring the picture (see below). Despite this potential obstacle, studies on the same trait (e.g. grooming) in distinct $A$. mellifera populations sometimes showed comparable results (Arechavaleta-Velasco et al., 2012; Bourgeois et al., 2015).

Despite tremendous efforts to homogenise protocols (Dietemann et al., 2013; Human et al., 2013), diverse methods and phenotyping protocols are still employed to study a given honey bee trait. Moreover, results of transcriptomic studies vary according to the choice of samples used, including the number of individuals, caste, age and organs or tissue of the bees. Altogether, given these technical differences, it is perhaps not surprising to observe so little overlap between studies.

Ideally, high-throughput analyses performed on the same biological system should yield similar outcomes. However, Fig. 5 clearly shows that this is not the case. Other reports also document that across 'omics fields, variation in results is considerable and transcript and protein abundance often do not correlate (Gygi et al., 1999; Liu et al., 2016). For example, studies on the same trait, population, and with the same sampling protocols, have given very different results, possibly due to differences in genotyping technologies, from QTL mapping using hundreds of microsatellites to high-throughput sequencing using thousands of SNPs (Behrens et al., 2011; Conlon et al., 2018). Altogether, this illustrates the clear need for homogenization and replication in order to tackle biological questions in ways that are less condition-dependent.

\subsection{Molecular mechanisms involved in Varroa resistance by honey bees}

Although there is a poor overlap of specific molecular markers between studies, the functions associated with such genetic markers and enriched in the different studies show some consistency between the different populations phenotyped. Notably, functions related to neural sensitivity, signal transmission, sensory perception, olfaction, transporter activity, metabolic process and oxidative phosphorylation have been found to be related to VSH and HYG in several studies (Supplementary Tables S2-S4). This suggests that neuronal functions and olfactory pathways play a key role in shaping the behavioural resistance to varroa, very likely via enhanced abilities to detect infested brood. This consistency, despite strong differences in methodologies (molecular techniques, targeted tissues, bee populations, etc.) is quite remarkable and demonstrates the strong link between those biological functions and varroa resistance related to VSH and HYG.

We performed an enrichment analysis on the 159 DEGs identified in at least two different transcriptomic studies of varroa resistance (using DAVID, Huang et al., 2009), but no molecular function and biological process was found to be significantly enriched, partly due to the relatively low number of DEGs (118 annotated) but also the different tissues that were analysed. However, investigating these 159 DEGs, five encode ion channel proteins (GB49268 - glutamate receptor ionotropic kainate 2, GB50159 - neuronal acetylcholine receptor subunit alpha-10, GB42728 - sodium channel protein paralytic, GB51897 - cacophony, GB50377 - two pore 
potassium channel protein sup-9) and six function as oxidoreductase (GB49380 - fatty acyl-CoA reductase 1, GB53651 - nitric oxide synthase, GB49878 - probable cytochrome P450 6a14, GB51356 cytochrome P450 4G11, GB41212 - laccase-5, GB50627 - putative fatty acyl-CoA reductase). Ion channel proteins are interesting since they are located on the membrane of excitable cells (neuron, muscle) and therefore likely involved in behavioural modulation. The prevalence of oxidoreductases suggests a different energy regulation in analysed tissues (including neurons and muscles). The roles of metabolic processes and oxidative phosphorylation in regulating HYG are not known, but are possibly linked to the differential modulation of neuron and muscle activity (Hall, 2012).

\subsection{Challenges implementing MAS}

The extremely high rates of genetic recombination in the honey bee is both a hindrance and a benefit for identifying genetic markers (Beye et al., 2006; Wallberg et al., 2015). The frequent recombination means that non-causal SNPs will quickly become dissociated from the phenotype. But in rare cases, it could lead to selection for an alternative causative allele. For example, Tsuruda et al. (2012) used a mapping population of VSH $\mathrm{x}$ unselected Italian bees to identify one major QTL that was associated with VSH behaviour. When VSH activity and the causative alleles at a key SNP were examined later in Russian stock, the allele that was associated with high VSH activity in the original mapping population was instead associated with lower activity in the Russian population (Kirrane et al., 2015). As the authors indicate, this suggests that a stable recombination event occurred after the two populations diverged. If MAS had been employed using this allele, VSH would theoretically be enriched in one stock and suppressed in the other. This example illustrates well that large scale allelic frequency studies combined with mechanistic confirmation or validating selective breeding experiments will be necessary to identify allelic markers that are reliable enough to be used in MAS across stocks. Although challenging, mechanistic confirmation or validating selective breeding experiments in diverse populations will be necessary to identify allelic markers that are reliable enough to be used in MAS across stocks. However, it is possible that such a 'one size fits all' marker does not exist, if different populations of honey bees are undergoing divergent evolution of varroa resistance traits - an idea that is consistent with the generally poor overlap between 'omics studies discussed above.
Once causal genetic markers for varroa resistance are identified, genetic testing has the advantage of being inexpensive and reliable; therefore, it is an ideal commercial diagnostic test. However, proteomic and transcriptomic technology is becoming increasingly robust and cost-effective, while not suffering from recombinationbased signal decay over time. A collaboration of Canadian researchers has developed a biomarker panel of 13 proteins (nine associated with HYG, two with VSH, and two with grooming), and have demonstrated that using these proteins for MAS yields resistance to varroa that is comparable to the leading field selection methods (Guarna et al., 2017). While this study validated biomarker efficacy in an independent population of $A$. mellifera, selection based on expression markers may still be sensitive to extraneous environmental variables. Grooming aptitude, for example, is known to vary with temperature (Currie and Tahmasbi, 2008), and so might its underlying transcript and protein markers.

\subsection{Odorant binding proteins (OBPs): a case study to illustrate the poor agreement of expression patterns across HYG studies}

Several studies have investigated differential expression patterns in honey bees from hygienic and non-hygienic colonies. Olfaction is widely agreed to be a key biological process enabling HYG (Gramacho and Spivak, 2003; Chakroborty et al., 2015): hygienic honey bees are better at odorant discrimination tasks and detect disease odorants at lower thresholds than nonhygienic honey bees. OBPs are thought to aid in peripheral odorant detection by binding and transporting odorant molecules from the antennal pore to the olfactory receptor neurons, but their expression in non-olfactory tissues suggests they have diverse biological roles. Many differential expression studies involving hygienic and non-hygienic honey bees have identified OBPs within the lists of significant genes and proteins. However, there is a huge diversity in the specific OBPs that have been identified (Table 4). For example, Gempe et al. (2016) found that OBP1 and OBP21 are downregulated in hygienic worker brains, but Boutin et al. (2015) and Scannapieco et al. (2017) found that OBP4 is downregulated in brains and heads. If there is one conserved molecular mechanism for HYG, the researchers theoretically should have identified the same OBPs even across geographically isolated populations. Similar inconsistencies are observed for gene expression in antennae of high and low VSH bees (Mondet et al., 2015; Hu et al., 2017). Some studies examined different tissues across which the same gene is differentially regulated. Confounding factors such as

Table 4

Significantly differentially expressed odorant binding proteins across studies: an illustrative example.

\begin{tabular}{|c|c|c|c|c|c|c|}
\hline Gene & Accession number (s) & Trait & Detection method & Tissue & Direction of regulation & Reference \\
\hline obp1 & GB55593, GB11135 & HYG & Microarray & Brain & Down & Gempe et al. (2016) \\
\hline obp1 & GB55593, GB11135 & HYG & QTL & - & - & Oxley et al. (2010) \\
\hline obp3 & GB53371, GB30242 & VSH & Microarray & Brain & Down & Le Conte et al. (2011) \\
\hline obp3 & GB53371, GB30242 & VSH & RNA-seq & Antennae & Up & Mondet et al. (2015) \\
\hline obp3 & GB53371, GB30242 & HYG & RT-qPCR & Head & Down & Scannapieco et al. (2017) \\
\hline obp3 & GB53371, GB30242 & GRM & Proteomics & Antennae & Up & Guarna et al. (2017) \\
\hline obp4 & GB53372, GB13587 & HYG & RNA-seq & Brain & Down & Boutin et al. (2015) \\
\hline obp4 & GB53372, GB13587 & HYG & RT-qPCR & Head & Down & Scannapieco et al. (2017) \\
\hline obp14 & GB46223 & VSH & RNA-seq & Antennae & Up & Mondet et al. (2015) \\
\hline obp14 & GB46223 & VSH & Proteomics & Brain & Up & Hu et al. (2016) \\
\hline obp15 & GB46224 & VSH & Proteomics & Antennae & Down & Hu et al. (2016) \\
\hline obp16 & GB46225, GB16826 & HYG & Proteomics & Antennae & Up & Guarna et al. (2017) \\
\hline obp17 & GB46226, GB11092 & VSH & Proteomics & Antennae & Up & Hu et al. (2016) \\
\hline obp18 & GB46227 & HYG, VSH & Proteomics & Antennae & Up & Hu et al. (2016); Guarna et al. (2017) \\
\hline obp18 & GB46227 & VSH & Proteomics & Brain & Up & Hu et al. (2016) \\
\hline obp18 & GB46227 & VSH & Proteomics & Hemolymph & Down & Hu et al. (2016) \\
\hline$o b p 21$ & GB46230, GB15460 & HYG & Microarray & Brain & Up & Gempe et al. (2016) \\
\hline
\end{tabular}

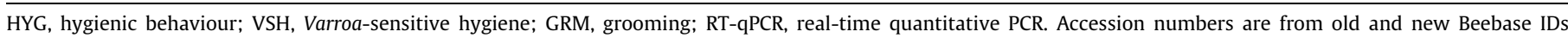
(hymenopteragenome.org, metazoan.ensembl.org). 
phenotyping techniques and instrumentation make it difficult to draw conclusions about why these OBP inconsistencies exist.

Other experimental evidence suggests that there could be multiple olfactory mechanisms underlying HYG. For example, phenethyl acetate acted as a strong HYG inducer in one population (Swanson et al., 2009), but not in another (McAfee et al., 2017b). It could be that honey bees with distinct genetic origins have low response thresholds to different disease odorants, detecting any one of which could be sufficient to elicit the behaviour. This is consistent with the idea that different odorant reception mechanisms, which enable detection of different disease odorant molecules, could underlie the same behaviour.

\section{Research gaps and perspectives}

The results of the two literature surveys we conducted highlight a great diversity of issues and knowledge gaps regarding the study of varroa resistance in A. mellifera. These gaps can be grouped in two main categories: biological and methodological aspects.

\subsection{Biological aspects}

An elemental step in breeding for specific resistance or tolerance traits is to accurately characterize trait heritability. (Moritz, 1985; Harbo and Harris, 1999b; Danka et al., 2016). Some traits are highly heritable and may indeed provide sustainable solutions to control the mite. This may be the case for MNR, for which empirical evidence has demonstrated high inheritance via drones or queens (Moritz, 1985; Harbo and Harris, 1999a; Danka et al., 2016; Locke, 2016). However, MNR expression within surviving colonies seems complex, as workers from the same subfamilies do not exhibit the trait homogeneously (Beaurepaire et al., 2019a). Generally, the exact inheritance mechanisms behind the different resistance and tolerance traits, as well as the extent of the variation of these mechanisms across individuals, subfamilies, colonies, and populations, are unknown.

Disease resistance and tolerance traits can act at several levels of social organization in honey bees (Kurze et al., 2016), and colony level expression is often overlooked. Honey bees live in complex, crowded societies, where nests are typically headed by one queen and contain a small fraction of reproductive drones, but the whole colony (the 'super-organism') is considered to be the unit of selection (Wheeler, 1928; Seeley, 1989). Due to this simplified view, important traits exhibited by individual workers are often overlooked (Moritz and Crewe, 2018). In fact, given honey bees' mating system, where queens typically mate with over a dozen unrelated drones (Tarpy et al., 2004), colonies are composed of a diversity of worker subfamilies with distinct genotypes that may differ in their responses to stressors (Page and Robinson, 1991). Moreover, within the same subfamily, individuals with identical genotypes may respond differently to parasites and pathogens, according to conditions such as age or environmental factors (Roberts and Hughes, 2014; Dalmon et al., 2019). There is a general consensus that intra-colonial diversity is highly adaptive for the expression of parasite resistance and tolerance, as it may buffer the colony level response threshold (Tarpy, 2003; Schmid-Hempel, 2011). However, more field studies are needed to confirm the effects of this high colony level genetic diversity and the relative expression of varroa resistance traits in the different subfamilies of honey bee colonies.

In addition to the intra-colonial level, the variation of traits between colonies in a given population is of great importance. Under natural conditions, the high admixture of drones in drone congregation areas provides opportunity for mixing of bee genotypes (Baudry et al., 1998; Harpur et al., 2019), and may facilitate the spread of resistance traits. In A. mellifera, drone brood is highly attractive to varroa mites (Le Conte et al., 1989). This attribute may enhance the selection of resistance in nature, since only the fittest males are capable of reaching a queen to mate in flight. Interestingly, studies investigating A. mellifera resistance traits in surviving populations often report multiple traits of significant value, and high variation across colonies (Locke, 2016). This suggests that it is the accumulation of multiple resistance traits that enables colonies to survive, and not one major trait.

\subsection{Methodological aspects}

Today, a great range of techniques and protocols have been developed to study resistance and tolerance in A. mellifera. Despite the general agreement of the research community to use specific techniques for investigating simple traits (e.g. opening cells to look for recapping), there is currently a lack of clarity on the precision and accuracy of the methods. In addition, the diversity of available techniques for a given trait generally grows with trait complexity. For instance, quantifying even something seemingly simple such as MPG in A. mellifera colonies can be very challenging. Several methods to infer the number of parasites infesting honey bee colonies currently exist: counting fallen mites on the bottom board, estimating phoretic mites using samples of adult bees, or counting mites in the brood (Dietemann et al., 2013; Gregorc and Sampson, 2019). Often, only one of these estimates is used to infer the total population of mites infesting a colony, as combining them is too time-consuming. However, due to fluctuations of brood and adult dynamics in space (e.g. across honey bee populations) and in time (e.g. between seasons), the ratio of mites and bees is constantly changing, and no single estimate can correctly predict entire parasite populations. Consequently, these estimates of mites in brood or on adult bees will not correctly assess the complete populations of parasites, unless they are combined with and/or used in parallel with models allowing for inference of spatiotemporal patterns of mite fluctuations (e.g. Calis et al., 1999). A better understanding of the mechanisms underlying each trait and the link between traits may help in finding proxies that could be used to facilitate phenotyping.

Even though the ability of honey bee colonies to survive varroa without mite control stands as the ultimate goal of varroa resistance and tolerance selection, using "survival" as the only phenotype may be dangerous since it may be dependent on many other factors besides varroa. Our literature review revealed that low MPG is a common downstream result in surviving populations. This highlights three important aspects for methodological development: (i) low MPG can result from many different traits operating alone or in combination; (ii) it can also be influenced by many environmental and beekeeping factors; and (iii) standing as a central feature of varroa-resistant populations, evaluating MPG may be the most robust phenotyping method to assess mite resistance. Future research should thus assess whether MPG can be used as a proxy to phenotype varroa resistance when used alone (such as in the Russian bees breeding program; Rinderer et al., 2001, 2005), or whether it needs to be used in combination with the phenotyping of one or several regulatory traits.

With a growing number of $A$. mellifera surviving populations reported (e.g. Kohl and Rutschmann, 2018; McMullan, 2018), there is a great need to simplify and standardize methods to confirming survivorship and characterize underlying mechanisms. To ensure the suitability of these methods, they should be rigorously tested across several honey bee populations and different environments. This could result in the development of a common breeding effort to solve the varroa problem across the globe, or determine that more local approaches have to be conducted. To date, the available data suggests that the latter is most likely, since adaptation to local environments seems to play a determining role in colony survival (Meixner et al., 2015). 


\subsection{Parasite adaptations might play a large role in host resistance or} tolerance

To date, studies on the resistance and tolerance to varroa have largely ignored the role of the parasite itself (Eliash and Mikheyev, 2020). This omission may be due to the fact that the introduced mite populations were considered pseudo-clonal due to the bottlenecks following host shifts and invasion of the parasite globally (Solignac et al., 2005), and therefore not likely to exhibit variation in complex traits. However, more recent evidence shows that Varroa spp. have shown that the mite populations are more genetically variable than previously thought (Robertson et al., 2014; Beaurepaire et al., 2015; Dynes et al., 2017; Dietemann et al., 2019), and that they can evolve swiftly in response to selective pressures such as acaricide treatments (Beaurepaire et al., 2017) in an arms race with their host (Beaurepaire et al., 2019b). Although clear links between these genetic findings and phenotypes in the field are currently missing, investigating the mite side of the story will help disentangle the role of the parasite traits in host resistance (Fries and Camazine, 2001; Seeley, 2017).

The relationship between $V$. destructor and $A$. mellifera still remains greatly unbalanced due to continuous human interference (Neumann and Blaquiere, 2016). This disequilibrium affects not only the honey bee, but also the mite populations for which collapsing colonies may represent a dead-end. Thus, an arms race between the host and the parasite should lead to an evolutionary equilibrium, where both species can perpetuate in more balanced ways (Thompson, 1994).

\section{Conclusions}

The resistance and tolerance mechanisms of honey bees that survive with varroa, whether acquired through natural selection or through selective breeding efforts, span a tremendous range of honey bee behaviour, individual immunity, population dynamics, and relationships with associated pathogens. Moreover, it is very likely that these mechanisms do not operate alone but may function in combination. The importance of specific adaptations may vary across environments. Future research should aim at understanding the potential links between traits, and why so little overlap is found in studies looking at molecular pathways underlying varroa resistance and tolerance. Such efforts will also help develop practical tools to assist selection programs to enhance varroa resistance and tolerance, either by offering molecular markers or by finding proxies of complex traits that could help with phenotyping colonies in the field. Phenotyping efforts and molecular maker development are complimentary approaches, and efficient development of MAS relies on the development of effective and reliable phenotyping tools.

The results of these analyses highlight the need to unify efforts in the research community, while presenting the most promising traits for future efforts in selective breeding for varroa-surviving bees.

\section{Acknowledgement}

This research was supported by a grant of The Persephone Charitable and Environmental Trust, Gibraltar.

\section{Appendix A. Supplementary data}

Supplementary data to this article can be found online at https://doi.org/10.1016/j.ijpara.2020.03.005.

\section{References}

Amdam, G.V., Hartfelder, K., Norberg, K., Hagen, A., Omholt, S.W., 2004. Altered physiology in worker honey bees (Hymenoptera: Apidae) infested with the mite Varroa destructor (Acari: Varroidae): a factor in colony loss during overwintering?. J. Econ. Entomol. 97 (3), 741-747.

Arechavaleta-Velasco, M.E., Alcala-Escamilla, K., Robles-Rios, C., Tsuruda, J.M., Hunt, G.J., 2012. Fine-scale linkage mapping reveals a small set of candidate genes influencing honey bee grooming behavior in response to Varroa mites. PLoS One 7, e47269.

Aumeier, P., Rosenkranz, P., Gonçalves, L.S., 1996. Defense mechanisms of honey bees against varroosis and brood diseases: comparison between Apis mellifera carnica and Africanized bees in Brazil. Apidologie 4 (27), 286-288. Ref. 4.

Aumeier, P., Rosenkranz, P., Gonçalves, L.S., 2000. A comparison of the hygienic response of Africanized and European (Apis mellifera carnica) honey bees to Varroa-infested brood in tropical Brazil. Genet. Mol. Biol. 23 (4), 787-791. Ref 2.

Aumeier, P., 2001. Bioassay for grooming effectiveness towards Varroa destructor mites in Africanized and Carniolan honey bees. Apidologie 32 (1), 81-90. Ref. 1.

Aumeier, P., Rosenkranz, P., Francke, W., 2002. Cuticular volatiles, attractivity of worker larvae and invasion of brood cells by Varroa mites. A comparison of Africanized and European honey bees. Chemoecology. 12(2), 65-75. Ref. 3.

Bahreini, R., Currie, R.W., 2015. The effect of queen pheromone status on Varroa mite removal from honey bee colonies with different grooming ability. Exp. Appl. Acarol. 66 (3), 383-397. Ref. 5.

Baudry, E., Solignac, M., Garnery, L., Gries, M., Cornuet, J., Koeniger, N., 1998 Relatedness among honeybees (Apis mellifera) of a drone congregation. Proc. Biol. Sci. B 265 (1409), 2009-2014.

Beaurepaire, A.L., Truong, T.A., Fajardo, A.C., Dinh, T.Q., Cervancia, C., Moritz, R.F. 2015. Host specificity in the honeybee parasitic mite, Varroa spp. in Apis mellifera and Apis cerana. PloS One 10, (8) e0135103.

Beaurepaire, A.L., Krieger, K.J., Moritz, R.F.A., 2017. Seasonal cycle of inbreeding and recombination of the parasitic mite Varroa destructor in honeybee colonies and its implications for the selection of acaricide resistance. Infect. Genet Evol. 50, 49-54.

Beaurepaire, A., Sann, C., Arredondo, D., Mondet, F., Le, Y., Conte, 2019a. Behavioral Genetics of the Interactions between Apis mellifera and Varroa destructor. Insects 10 (9), 299.

Beaurepaire, A.L., Moro, A., Mondet, F., Le Conte, Y., Neumann, P., Locke, B., 2019b. Population genetics of ectoparasitic mites suggest arms race with honeybee hosts. Sci. Rep. 9 (1), 1-9.

Behrens, D., Huang, Q., Geßner, C., Rosenkranz, P., Frey, E., Locke, B., Moritz, R.F.A. Kraus, F.B., 2011. Three QTL in the honey bee Apis mellifera L. suppress reproduction of the parasitic mite Varroa destructor. Ecol. Evol. 1 (4), 451-458.

Bergman, T.J., Beehner, J.C., 2015. Measuring social complexity. Anim. Behav. 103, 203-209.

Beye, M., Gattermeier, I., Hasselmann, M., Gempe, T., Schioett, M., Baines, J.F., Schlipalius, D., Mougel, F., Emore, C., Rueppell, O., Sirviö, A., 2006. Exceptionally high levels of recombination across the honey bee genome. Genome Res. 16 (11), 1339-1344.

Boecking, O., Rath, W., Drescher, W., 1993. Behavioral strategies of Apis mellifera and Apis cerana against Varroa jacobsoni. Int. J. Acarol. 19 (2), 173-177.

Boecking, O., Bienefeld, K., Drescher, W., 2000. Heritability of the Varroa-specific hygienic behaviour in honey bees (Hymenoptera: Apidae). J. Anim. Breed Genet. 117 (6), 417-424.

Boot, W.J., Calis, J.N., Beetsma, J., Hai, D.M., Lan, N.K., Van Toan, T., Minh, N.H., 1999 Natural selection of Varroa jacobsoni explains the different reproductive strategies in colonies of Apis cerana and Apis mellifera. Exp. Appl. Acarol. 23 (2), 133-144.

Bourgeois, A.L., Villa, J.D., Holloway, B., Danka, R.G., Rinderer, T.E., 2015. Molecular genetic analysis of tracheal mite resistance in honey bees. J. Apic. Res. 54 (4), 314-320.

Boutin, S., Alburaki, M., Mercier, P.L., Giovenazzo, P., Derome, N., 2015. Differential gene expression between hygienic and non-hygienic honeybee (Apis mellifera L.) hives. BMC Genomics 16 (1), 500.

Büchler, R., Berg, S., Le Conte, Y., 2010. Breeding for resistance to Varroa destructor in Europe. Apidologie 41, 393-408.

Calis, J.N.M., Fries, I., Ryrie, S.C., 1999. Population modelling of Varroa jacobsoni Oud. Apidologie 30, 111-124.

Carneiro, F.E., Torres, R.R., Strapazzon, R., Ramírez, S.A., Guerra Jr., J.C., Koling, D.F. Moretto, G., 2007. Changes in the reproductive ability of the mite Varroa destructor (Anderson e Trueman) in Africanized honey bees (Apis mellifera L.) (Hymenoptera: Apidae) colonies in southern Brazil. Neotrop. Entomol. 36(6) 949-952. Ref. 6.

Carreck, N., Neumann, P., 2010. Honey bee colony losses. J. Apic. Res. 49 (1), 1-6.

Chakroborty, N.K., Bienefeld, K., Menzel, R., 2015. Odor learning and odor discrimination of bees selected for enhanced hygienic behavior. Apidologie 46 (4), 499-514.

Cheruiyot, S.K., Lattorff, H.M.G., Kahuthia-Gathu, R., Mbugi, J.P., Muli, E., 2018. Varroa-specific hygienic behavior of Apis mellifera scutellata in Kenya. Apidologie. 49(4), 439-449. Ref. 7.

Conlon, B.H., Frey, E., Rosenkranz, P., Locke, B., Moritz, R.F., Routtu, J., 2018. The role of epistatic interactions underpinning resistance to parasitic Varroa mites in haploid honey bee (Apis mellifera) drones. J. Evol. Biol. 31 (6), 801-809. 
Conlon, B., Aurori, A., Giurgiu, A.-I., Kefuss, J., Dezmirean, D.S., Moritz, R.F.A., Routtu, J., 2019. A gene for resistance to the Varroa mite (Acari) in honey bee (Apis mellifera) pupae. Mol. Ecol.

Corrêa-Marques, M.H., De Jong, D., 1998. Uncapping of worker bee brood, a component of the hygienic behavior of Africanized honey bees against the mite Varroa jacobsoni Oudemans. Apidologie. 29(3), 283-289. Ref. 9.

Corrêa-Marques, M.H., De Jong, D., Rosenkranz, P., Gonçalves, L.S., 2002. Varroatolerant Italian honey bees introduced from Brazil were not more efficient in defending themselves against the mite Varroa destructor than Carniolan bees in Germany. Genet. Mol. Res. 1(2), 153-158. Ref. 8.

Crane, E., 1968. Beekeeping round the World: Mites infesting honeybees in Asia. Bee World 49 (3), 113-114.

Cremer, S., Armitage, S.A., Schmid-Hempel, P., 2007. Social immunity. Curr. Biol. 17 (16), 693-702

Currie, R., Tahmasbi, G., 2008. The ability of high-and low-grooming lines of honey bees to remove the parasitic mite Varroa destructor is affected by environmental conditions. Can. J. Zool. 86, 1059-1067.

Dalmon, A., Peruzzi, M., Le Conte, Y., Alaux, C., Pioz, M., 2019. Temperature-driven changes in viral loads in the honey bee Apis mellifera. J. Invertebr. Pathol. 160, 87-94.

Danka, R.G., Harris, J.W., Villa, J.D., Dodds, G.E., 2013. Varying congruence of hygienic responses to Varroa destructor and freeze-killed brood among different types of honeybees. Apidologie. 44(4), 447-457. Ref. 10.

Danka, R.G., Harris, J.W., Dodds, G.E., 2016. Selection of VSH-derived "Pol-line" honey bees and evaluation of their Varroa-resistance characteristics. Apidologie 47 (3), 483-490. Ref. 11.

de Guzman, L.I., Rinderer, T.E., Frake, A.M., 2008. Comparative reproduction of Varroa destructor in different types of Russian and Italian honey bee combs. Exp. Appl. Acarol. 44 (3), 227-238. Ref 12.

de Jong, D., Soares, A.E.E., 1997. An isolated population of Italian bees that has survived Varroa jacobsoni infestation without treatment for over 12 years. Am. Bee. J. 4, 742-745. Ref $13 .$.

Dekkers, J.C.M., Hospital, F., 2002. The use of molecular genetics in the improvement of agricultural populations. Nat. Rev. Genet. 3, 22-32.

Dietemann, V., Pflugfelder, J., Anderson, D., Charrière, J.D., Chejanovsky, N., Dainat B., de Miranda, J., Delaplane, K., Dillier, F., Fuch, S., Gallmann, P., Gauthier, L., Imdorf, A., Koeniger, N., Kralj, J., Meikle, W., Pettis, J., Rosenkranz, P., Sammataro, D., Smith, D., Yañez, O., Neumann, P., 2012. Varroa destructor: research avenues towards sustainable control. J. Apic. Res. 51 (1), 125-132.

Dietemann, V., Nazzi, F., Martin, S.J., Anderson, D.L., Locke, B., Delaplane, K.S. Wauquiez, Q., Tannahill, C., Frey, E., Ziegelmann, B., Rosenkranz, P., Ellis, J.D. 2013. Standard methods for varroa research. J. Apic. Res. 52 (1), 1-54.

Dietemann, V., Beaurepaire, A., Page, P., Yañez, O., Buawangpong, N. Chantawannakul, P., Neumann, P., 2019. Population genetics of ectoparasitic mites Varroa spp. Eastern and Western honey bees. Parasitology 146 (11), 1429-1439.

Doublet, V., Poeschl, Y. Gogol-Döring, A., Alaux, C., Annoscia, D., Aurori, C., Barribeau, S.M., Bedoya-Reina, O.C., Brown, M.J., Bull, J.C., Flenniken, M.L. 2017. Unity in defense: honeybee workers exhibit conserved molecular responses to diverse pathogens. BMC Genomics 18 (1), 207.

Dynes, T.L., De Roode, J.C., Lyons, J.I., Berry, J.A., Delaplane, K.S., Brosi, B.J., 2017. Fine scale population genetic structure of Varroa destructor, an ectoparasitic mite of the honey bee (Apis mellifera). Apidologie 48 (1), 93-101.

Eguaras, M., Marcangeli, J., Oppedisano, M., Fernandez, N., 1995. Mortality and reproduction of Varroa jacobsoni in resistant colonies of honey bees (Apis mellifera) in Argentina. Bee Sci. 3, 174-178.

Eliash, N., Mikheyev, A., 2020. Varroa mite evolution: A neglected aspect of worldwide bee collapses? Curr. Opin. Insect Sci.

Elsik, C.G., Tayal, A., Unni, D.R., Burns, G.W., Hagen, D.E., 2018. Hymenoptera genome database: using hymenopteramine to enhance genomic studies of hymenopteran insects. In: Eukaryotic Genomic Databases. Humana Press, New York, NY, pp. 513-556.

Fonio, E., Golani, I., Benjamini, Y., 2012. Measuring behavior of animal models: faults and remedies. Nat. Methods 9 (12), 1167

Fries, I., Huazhen, W., Wei, S., Jin, C.S., 1996. Grooming behavior and damaged mites (Varroa jacobsoni) in Apis cerana cerana and Apis mellifera ligustica. Apidologie 27 (1), 3-11.

Fries, I., Camazine, S., 2001. Implications of horizontal and vertical pathogen transmission for honey bee epidemiology. Apidologie 32 (3), 199-214.

Fries, I., Imdorf, A., Rosenkranz, P., 2006. Survival of mite infested (Varroa destructor) honey bee (Apis mellifera) colonies in a Nordic climate. Apidologie 37 (5), 564 570. Ref. 14.

Fries, I., Bommarco, R., 2007. Possible host-parasite adaptations in honey bees infested by Varroa destructor mites. Apidologie. 38 (6), 525-533. Ref. 15

Garrido, C., Rosenkranz, P., Paxton, R.J., Gonçalves, L.S., 2003. Temporal changes in Varroa destructor fertility and haplotype in Brazil. Apidologie. 34(6), 535-541. Ref. 16.

Gempe, T., Stach, S., Bienefeld, K., Otte, M., Beye, M., 2016. Behavioral and molecular studies of quantitative differences in hygienic behavior in honeybees. BMC Res. Notes 9(1), 474. Ref. Table 3.

Gramacho, K.P., Spivak, M., 2003. Differences in olfactory sensitivity and behavioral responses among honey bees bred for hygienic behavior. Behav. Ecol. Sociobiol. 54 (5), 472-479.

Gregorc, A., Sampson, B., 2019. Diagnosis of Varroa Mite (Varroa destructor) and sustainable control in honey bee (Apis mellifera) Colonies-A review. Diversity 11 (12), 243.
Grozinger, C.M., Robinson, G.E., 2015. The power and promise of applying genomics to honey bee health. Curr Opin. Insect Sci. 10, 124-132.

Guarna, M.M., Hoover, S.E., Huxter, E., Higo, H., Moon, K.M., Domanski, D, Bixby, M. E.F., Melathopoulos, A.P., Ibrahim, A., Peirson, M., Desai, S., Micholson, D., White, R., Borchers, C.H., Currie, R.W., Pernal, S.F., Desai, S., 2017. Peptide biomarkers used for the selective breeding of a complex polygenic trait in honey bees. Sci. Rep. 7(1), 8381. Ref. Table 3.

Guerra, M., 2000. Patterns of heterochromatin distribution in plant chromosomes. Genet. Molec. Biol. 23(4), 1029-1041. Ref. 17

Guzmán-Novoa, E., Vandame, R., Arechavaleta, M.E., 1999. Susceptibility of European and Africanized honey bees (Apis mellifera L.) to Varroa jacobsoni Oud. in Mexico. Apidologie. 30(2-3), 173-182. Ref. 18.

Guzmán-Novoa, E., Emsen, B., Unger, P., Espinosa-Montaño, L.G., Petukhova, T., 2012. Genotypic variability and relationships between mite infestation levels, mite damage, grooming intensity, and removal of Varroa destructor mites in selected strains of worker honey bees (Apis mellifera L.). J. Invertebr. Pathol. 110 (3), 314-320. Ref. 19.

Gygi, S.P., Rochon, Y., Franza, B.R., Aebersold, R., 1999. Correlation between protein and mRNA abundance in yeast. Mol. Cell. Biol. 19 (3), 1720-1730.

Hall, K.D., 2012. Modeling metabolic adaptations and energy regulation in humans. Annu. Rev. Nutr. 32 (1), 35-54.

Harbo, J.R., Hoopingarner, R.A., 1997. Honey Bees (Hymenoptera: Apidae) in the United States that express Resistance to Varroa jacobsoni (Mesostigmata: Varroidae). J. Econ. Entomol. 90(4), 893-898. Ref. 21.

Harbo, J.R., Harris, J.W., 1999a. Selecting honey bees for resistance to Varroa jacobsoni. Apidologie 30 (2-3), 183-196.

Harbo, J.R., Harris, J.W., 1999b. Heritability in honey bees (Hymenoptera: Apidae) of characteristics associated with resistance to Varroa jacobsoni (Mesostigmata: Varroidae). J. Econ. Entomol. 92 (2), 261-265.

Harbo, J.R., Harris, J.W., 2003. An evaluation of commercially produced queens that have the SMR trait. Am. Bee J. 143 (3), 213-216.

Harbo, J.R., Harris, J.W., 2005. Suppressed mite reproduction explained by the behaviour of adult bees. J. Apic. Res. 44 (1), 21-23. Ref. 20.

Harpur, B.A., Guarna, M.M., Huxter, E., Higo, H., Moon, K.M., Hoover, S.E., Ibrahim, A., Melathopoulos, A.P., Desai, S., Currie, R.W., Pernal, S.F., Foster, L.J., Zayed, A., Pernal, S.F., 2019. Integrative genomics reveals the genetics and evolution of the honey bee's social immune system. Genome Biol. Evol. 11 (3), 937-948.

Harris, J.W., Rinderer, T.E., 2004. Varroa resistance of hybrid ARS Russian honey bees. Am. Bee J. 144(10), 797-800. Ref. 24.

Harris, J.W., 2007. Bees with Varroa Sensitive Hygiene preferentially remove mite infested pupae aged < five days post capping. J. Apic. Res. 46 (3), 134-139.

Harris, J.W., Danka, R.G., Villa, J.D., 2010. Honey bees (Hymenoptera: Apidae) with the trait of Varroa sensitive hygiene remove brood with all reproductive stages of Varroa mites (Mesostigmata: Varroidae). Ann. Entomol. Soc. Am. 103(2), 146-152. Ref. 22.

Harris, J.W., Danka, R.G., Villa, J.D., 2012. Changes in infestation, cell cap condition, and reproductive status of Varroa destructor (Mesostigmata: Varroidae) in brood exposed to honey bees with Varroa sensitive hygiene. Ann. Entomol. Soc Am. 105(3), 512-518. Ref. 23.

Hu, H., Bienefeld, K., Wegener, J., Zautke, F., Hao, Y., Feng, M., Han, B., Fang, Y., Wubie, A.J., Li, J., 2016. Proteome analysis of the hemolymph, mushroom body, and antenna provides novel insight into honeybee resistance against Varroa infestation. J. Proteome. Res. 15(8), 2841-2854. Ref. Table 3.

Huang, D., Sherman, B., Lempicki, R., 2009. Systematic and integrative analysis of large gene lists using DAVID bioinformatics resources. Nat. Protoc. 4, 4457.

Human, H., Brodschneider, R., Dietemann, V., Dively, G., Ellis, J.D., Forsgren, E., Fries, I., Hatjina, F., Hu, F., Jaffé, R., Jensen, A.B., Köhler, A., Magyar, J.P., Özkýrým, A., Pirk, C.W.W., Rose, R., Strauss, U., Tanner, G., Tarpy, D.R., van der Steen, J.J.M., Vaudo, A., Vejsnæs, F., Wilde, J., Williams, G.R., Zheng, H., 2013. Miscellaneous standard methods for Apis mellifera research. J. Apic. Res. 52 (4), 1-53.

Ibrahim, A., Spivak, M., 2006. The relationship between hygienic behavior and suppression of mite reproduction as honey bee (Apis mellifera) mechanisms of resistance to Varroa destructor. Apidologie 37(1), 31-40. Ref. 25

Ibrahim, A., Reuter, G.S., Spivak, M., 2007. Field trial of honey bee colonies bred for mechanisms of resistance against Varroa destructor. Apidologie 38(1), 67-76. Ref. 26.

Johnson, R.M., Pollock, H.S., Berenbaum, M.R., 2009. Synergistic interactions between in-hive miticides in Apis mellifera. J. Econ. Entomol. 102 (2), 474-479.

Johnson, R.M., Dahlgren, L., Siegfried, B.D., Ellis, M.D., 2013. Acaricide, fungicide and drug interactions in honey bees (Apis mellifera). PloS One 8, (1) e54092.

Kefuss, J., Vanpoucke, J., De Lahitte, J.D., Ritter, W., 2004. Varroa tolerance in France of intermissa bees from Tunisia and their naturally mated descendants: 19932004. Am. Bee. J. 144(7), 563-568. Ref. 27.

Kefuss, J., Vanpoucke, J., Bolt, M., Kefuss, C., 2015. Selection for resistance to Varroa destructor under commercial beekeeping conditions. J. Apic. Res. 54(5), 563576. Ref. 28

Kersey, P.J., Allen, J.E., Allot, A., Barba, M., Boddu, S., Bolt, B.J., Carvalho-Silva, D., Christensen, M. Davis, P. Grabmueller, C. Kumar, N. Liu, Z, Maurel, T. Moore, B., McDowall, M.D., Maheswari, U., Naamati, G., Newman, V., Ong, C.K., Paulini, M., Pedro, H., Perry, E., Russell, M., Sparrow, H., Tapanari, E., Taylor, K., Vullo, A., Williams, G., Zadissia, A., Olson, A., Stein, J., Wei, S., Tello-Ruiz, M., Ware, D., Luciani, A., Potter, S., Finn, R.D., Urban, M., Hammond-Kosack, K.E., Bolser, D.M., De Silva, N., Howe, K.L., Langridge, N., Maslen, G., Staines, D.M., Yates, A., 2018. Ensembl genomes 2018: an integrated omics infrastructure for non-vertebrate species. Nucleic Acids Res. 46, 802-808. 
Khongphinitbunjong, K., de Guzman, L.I., Rinderer, T.E., Tarver, M.R., Frake, A.M., Chen, Y., Chantawannakul, P., 2016. Responses of Varroa-resistant honey bees (Apis mellifera L.) to deformed wingvirus. J. Asia Pac. Entomol. 19(4), 921-927. Ref. 29.

Kirrane, M.J., de Guzman, L.I., Holloway, B., Frake, A.M., Rinderer, T.E., Whelan, P.M., 2015. Phenotypic and genetic analyses of the Varroa sensitive hygienic trait in Russian honey bee (Hymenoptera: Apidae) colonies. PloS One. 10(4), e0116672. Ref. 30.

Kirrane, M.J., De Guzman, L.I., Whelan, P.M., Frake, A.M., Rinderer, T.E., 2018. Evaluations of the removal of Varroa destructor in Russian honey bee colonies that display different levels of Varroa sensitive hygienic activities. J. Insect Behav. 31(3), 283-297. Ref. 31.

Kohl, P.L., Rutschmann, B., 2018. The neglected bee trees: European beech forests as a home for feral honey bee colonies. PeerJ 6, e4602.

Kruitwagen, A., van Langevelde, F., van Dooremalen, C., Blacquière, T., 2017. Naturally selected honey bee (Apis mellifera) colonies resistant to Varroa destructor do not groom more intensively. J. Apic. Res. 56(4), 354-365. Ref. 32. Kulikov, N.S., 1965. Varrosis of bees. Pchelovodstvo 85 (11), 15-16.

Kulinčević, J.M., Rinderer, T.E., Mladjan, V.J., Buco, S.M., 1992. Five years of bidirectional genetic selection for honey bees resistant and susceptible to Varroa jacobsoni. Apidologie 23(5), 443-452. Ref. 33.

Kurze, C., Routtu, J., Moritz, R.F., 2016. Parasite resistance and tolerance in honeybees at the individual and social level. Zoology 119 (4), 290-297.

Lattorff, H.M.G., Buchholz, J., Fries, I., Moritz, R.F.A., 2015. A selective sweep in a Varroa destructor resistant honeybee (Apis mellifera) population. Infect. Genet. Evol. 31, 169-176. https://doi.org/10.1016/j.meegid.2015.01.025.

Yves, Le Conte, Ellis, M., Ritter, W., 2010. Varroa mites and honey bee health: can Varroa explain part of the colony losses? Apidologie 41, 353-363. https://doi. org/10.1051/apido/2010017.

Leclercq, G., Blacquière, T., Gengler, N., Francis, F., 2018. Hygienic removal of freezekilled brood does not predict Varroa-resistance traits in unselected stocks. J. Apic. Res. 57 (2), 292-299.

Le, Y., Conte, Arnold, G., Trouiller, J., Masson, C., Chappe, B., Ourisson, G., 1989. Attraction of the parasitic mite Varroa to the drone larvae of honey bees by simple aliphatic esters. Science 245 (4918), 638-639.

Le, Y., Conte, Bruchou, C., Benhamouda, K., Gauthier, C., Cornuet, J.M., 1994. Heritability of the queen brood post-capping stage duration in Apis mellifera mellifera L. Apidologie 25 (6), 513-519.

Le Conte, Y., De Vaublanc, G., Crauser, D., Jeanne, F., Rousselle, J.C., Bécard, J.M., 2007. Honey bee colonies that have survived Varroa destructor. Apidologie 38 (6), 566-572. Ref. 34.

Le Conte, Y., Alaux, C., Martin, J.F., Harbo, J.R., Harris, J.W., Dantec, C., Sévérac, D., Cors-Arteil, S., Navajas, M., 2011. Social immunity in honeybees (Apis mellifera): transcriptome analysis of varroa-hygienic behaviour. Insect Mol. Biol. 20(3), 399-408. Ref. 35.

Lin, Z., Qin, Y., Page, P., Wang, S., Li, L., Wen, Z., Hu, F., Neumann, P., Zheng, H., Dietemann, V., 2018. Reproduction of parasitic mites Varroa destructor in original and new honeybee hosts. Ecol. Evol. 8 (4), 2135-2145.

Liu, Y., Yan, L., Li, Z., Huang, W.F., Pokhrel, S., Liu, X., Su, S., 2016. Larva-mediated chalkbrood resistance-associated single nucleotide polymorphism markers in the honey bee Apis mellifera. Insect Mol. Biol. 25 (3), 239-250.

Locke, B., Forsgren, E., Fries, I., de, J.R., Miranda, 2012. Acaricide treatment affects viral dynamics in Varroa destructor-infested honey bee colonies via both host physiology and mite control. Appl. Environ. Microbiol. 78 (1), 227-235.

Locke, B., Le Conte, Y., Crauser, D., Fries, I., 2012b. Host adaptations reduce the reproductive success of Varroa destructor in two distinct European honey bee populations. Ecol. Evol. 2(6), 1144-1150. Ref. 38.

Locke, B., Forsgren, E., de Miranda, J.R., 2014. Increased tolerance and resistance to virus infections: a possible factor in the survival of Varroa destructor-resistant honey bees (Apis mellifera). PloS One. 9(6), e99998. Ref. 37.

Locke, B., 2016. Natural Varroa mite-surviving Apis mellifera honeybee populations. Apidologie, 1-16.

Loftus, J.C., Smith, M.L., Seeley, T.D., 2016. How honey bee colonies survive in the wild: testing the importance of small nests and frequent swarming. PloS One. 11(3), e0150362. Ref. 39.

Martin, S.J., 1994. Ontogenesis of the mite Varroa jacobsoni Oud. in worker brood of the honeybee Apis mellifera L. under natural conditions. Experim. Appl. Acarol. 18, 87-100.

Martin, S., Hogarth, A., Van, J., Breda, Perrett, J., 1998. A scientific note on Varroa jacobsoni Oudemans and the collapse of Apis mellifera L. colonies in the United Kingdom. Apidologie 29 (4), 369-370.

Martin, C., Provost, E., Roux, M., Bruchou, C., Crauser, D., Clement, J.L., Le Conte, Y., 2001. Resistance of the honey bee, Apis mellifera to the acarian parasite Varroa destructor: behavioural and electroantennographic data. Physiol. Entomol. 26 (4), 362-370. Ref. 41.

Martin, S.J., Highfield, A.C., Brettell, L., Villalobos, E.M., Budge, G.E., Powell, M., Nikaido, S., Schroeder, D.C., 2012. Global honey bee viral landscape altered by a parasitic mite. Science. 336 (6086), 1304-1306.

Moritz, R.F.A., Crewe, R.M., 2018. The Dark Side of the Hive - the Evolution of the Imperfect Honey bee. Oxford University Press.

McAfee, A., Collins, T.F., Madilao, L.L., Foster, L.J., 2017a. Odorant cues linked to social immunity induce lateralized antenna stimulation in honey bees (Apis mellifera L.). Sci. Rep. 7, 46171.

McAfee, A., Chan, Q.W., Evans, J., Foster, L.J., 2017b. A Varroa destructor protein atlas reveals molecular underpinnings of developmental transitions and sexual differentiation. Mol. Cell. Proteomics 16 (12), 2125-2137.
McAfee, A., Chapman, A., Iovinella, I., Gallagher-Kurtzke, Y., Collins, T.F., Higo, H., Madilao, L.L., Pelosi, P., Foster, L.J., 2018. A death pheromone, oleic acid, triggers hygienic behavior in honey bees (Apis mellifera L.). Sci. Rep. 8 (1), 1-13.

McMullan, J., 2018. Adaptation in honey bee (Apis mellifera) colonies exhibiting tolerance to Varroa destructor in Ireland. Bee World. 95(2), 39-43. Ref. 40.

Medina, L.M., Martin, S.J., 1999. A comparative study of Varroa jacobsoni reproduction in worker cells of honey bees (Apis mellifera) in England and Africanized bees in Yucatan, Mexico. Experim. Appl. Acarol. 23(8), 659-667. Ref. 42.

Meixner, M.D., Kryger, P., Costa, C., 2015. Effects of genotype, environment, and their interactions on honey bee health in Europe. Curr. Opin. Insect. Sci. 10, $177-184$.

Message, D., Goncalves, L.S., 1995. Effect of size of worker brood cells of Africanized honey bees on infestation and replication of the ectoparasitic mite Varroa jacobsoni. Apidologie. 26, 381-386. Ref. 43.

Meyers, B.C., Kaushik, S., Nandety, R.S., 2005. Evolving disease resistance genes Curr. Opin. Plant Biol. 8 (2), 129-134.

Milani, N., 1999. The resistance of Varroa jacobsoni Oud. to acaricides. Apidologie 30 (2-3), 229-234.

Milani, N., Della Vedova, G., Nazzi, F., 2004. (Z)-8-heptadecene reduces the reproduction of Varroa destructor in brood cells. Apidologie 35 (3), 265-273.

Mondet, F., Conte, Y.L., 2014. Parasites. In: Ritter, W. (Ed.), Bee health and Veterinarians. OIE, pp. 131-141.

Mondet, F., de, J.R., Miranda, Kretzschmar, A., Le, Y., Conte, Mercer, A.R., 2014. On the front line: quantitative virus dynamics in honeybee (Apis mellifera L.) colonies along a new expansion front of the parasite Varroa destructor. PLoS Pathog. 10, (8) e1004323.

Mondet, F., Alaux, C., Severac, D., Rohmer, M., Mercer, A.R., Le Conte, Y., 2015 Antennae hold a key to Varroa-sensitive hygiene behaviour in honey bees. Sci. Rep. 5, 10454. Ref. Table 3.

Mondet, F., Kim, S.H., De Miranda, J.R., Beslay, D., Le Conte, Y., Mercer, A.R., 2016 Specific cues associated with honey bee social defence against Varroa destructor infested brood. Sci. Rep. 6, 25444.

Mondragón, L., Spivak, M., Vandame, R., 2005. A multifactorial study of the resistance of honeybees Apis mellifera to the mite Varroa destructor over one year in Mexico. Apidologie 36(3), 345-358. Ref. 44.

Mondragón, L., Martin, S., Vandame, R., 2006. Mortality of mite offspring: a major component of Varroa destructor resistance in a population of Africanized bees. Apidologie 37(1), 67-74. Ref. 45.

Mordecai, G.J., Brettell, L.E., Martin, S.J., Dixon, D., Jones, I.M., Schroeder, D.C., 2016 Superinfection exclusion and the long-term survival of honey bees in Varroainfested colonies. ISME J. 10(5), 1182. Ref. 46.

Moretto, G., Gonçalves, L.S., De Jong, D., 1995. Analysis of the F1 generation, descendants of Africanized bee colonies with differing defense abilities against the mite Varroa jacobsoni. Rev. Bras. Genet. 18. 177-177.

Moritz, R.F.A., Hänel, H., 1984. Restricted development of the parasitic mite Varroa jacobsoni Oud. in the Cape honeybee Apis mellifera capensis. J. Appl. Entomol. 97(1-5), 91-95. Ref. 48.

Moritz, R.F.A., 1985. Heritability of the postcapping stage in Apis mellifera and its relation to varroatosis resistance. J. Hered. 76(4), 267-270. Ref. 47.

Moritz, R.F.A., Mautz, D., 1990. Development of Varroa jacobsoni in colonies of Apis mellifera capensis and Apis mellifera carnica. Apidologie. 21(1), 53-58. Ref. 49.

Navajas, M., Migeon, A., Alaux, C., Martin-Magniette, M.L., Robinson, G.E., Evans, J.D. S. Cros-Arteil, Crauser D., Le Conte, Y., 2008. Differential gene expression of the honey bee Apis mellifera associated with Varroa destructor infection. BMC Genomics 9(1), 301. Ref. 50.

Nazzi, F., Milani, N., 1996. The presence of inhibitors of the reproduction of Varrod jacobsoni Oud. (Gamasida: Varroidae) in infested cells. Exp. Appl. Acarol. 20 (11), 617-623.

Nazzi, F., Milani, N., Della Vedova, G., 2004. A semiochemical from larval food influences the entrance of Varroa destructor into brood cells. Apidologie 35 (4), 403-410.

Neumann, P., Blacquière, T., 2016. The Darwin cure for apiculture? Natural selection and managed honeybee health. Evol. Appl. 10 (3), 226-230.

Nganso, B.T., Fombong, A.T., Yusuf, A.A., Pirk, C.W., Stuhl, C., Torto, B., 2018. Low fertility, fecundity and numbers of mated female offspring explain the lower reproductive success of the parasitic mite Varroa destructor in African honeybees. Parasitology 145(12), 1633-1639. Ref. 51.

Oddie, M.A., Dahle, B., Neumann, P., 2017. Norwegian honey bees surviving Varroa destructor mite infestations by means of natural selection. PeerJ 5, e3956. Ref. 52.

Oddie, M., Büchler, R., Dahle, B., Kovacic, M., Le Conte, Y., Locke, B., de Miranda, J.R. Mondet, F., Neumann, P., 2018a. Rapid parallel evolution overcomes global honey bee parasite. Sci. Rep. 8(1), 7704. Ref. 53.

Oddie, M., Dahle, B., Neumann, P., 2018b. Reduced postcapping period in honey bees surviving Varroa destructor by means of natural selection. Insects. 9(4), 149 Ref. 54.

Oddie, M.A., Neumann, P., Dahle, B., 2019. Cell size and Varroa destructor mite infestations in susceptible and naturally-surviving honeybee (Apis mellifera) colonies. Apidologie 50 (1), 1-10.

Oldroyd, B.P., 1999. Coevolution while you wait: Varroa jacobsoni, a new parasite of western honeybees. Trends Ecol. Evol. 14 (8), 312-315.

Oxley, P.R., Spivak, M., Oldroyd, B.P., 2010. Six quantitative trait loci influence task thresholds for hygienic behaviour in honeybees (Apis mellifera). Mol. Ecol. 19 (7), 1452-1461. Ref. Table 3. 
Page, R.E., Robinson, G.E., 1991. The genetics of division of labour in honey bee colonies. Adv. Insect Physiol. 23, 117-169.

Page, P., Lin, Z., Buawangpong, N., Zheng, H., Hu, F., Neumann, P., Chantawannakul, P., Dietemann, V., 2016. Social apoptosis in honey bee superorganisms. Sci. Rep. 6, 27210.

Panziera, D., van Langevelde, F., Blacquière, T., 2017. Varroa sensitive hygiene contributes to naturally selected varroa resistance in honey bees. J. Apic. Res. 56 (5), 635-642. Ref. 55

Peng, Y.S., Fang, Y., Xu, S., Ge, L., 1987. The resistance mechanism of the Asian honey bee, Apis cerana Fabr., to an ectoparasitic mite, Varroa jacobsoni Oudemans. J. Invertebr. Pathol. 49 (1), 54-60.

Pinto, M.A., Muñoz, I., Chávez-Galarza, J., De la Rúa, P., 2012. The Atlantic side of the Iberian Peninsula: a hot-spot of novel African honey bee maternal diversity. Apidologie. 43(6), 663-673. Ref. 56.

Popova, M., Reyes, M., Le Conte, Y., Bankova, V., 2014. Propolis chemical composition and honeybee resistance against Varroa destructor. Nat. Prod. Res. 28(11), 788-794. Ref. 57.

Ramsey, S.D., Ochoa, R., Bauchan, G., Gulbronson, C., Mowery, J.D., Cohen, A., Lim, D. Joklik, J., Cicero, J.M., Ellis, J.D., Hawthorne, D., VanEngelsdorp, D., 2019. Varroa destructor feeds primarily on honey bee fat body tissue and not hemolymph. Proc. Natl. Acad. Sci. U.S.A. 116 (5), 1792-1801.

Rath, W., Drescher, W., 1990. Response of Apis cerana Fabr. towards brood infested with Varroa jacobsoni Oud. and infestation rate of colonies in Thailand. Apidologie 21, 311-321.

Rinderer, T.E., Delatte, G.T., de Guzman, L.I., Williams, J., Stelzer, J.A., Kuznetsov, V.N, 1999. Evaluations of the Varroa-resistance of honey bees imported from fareastern Russia. Am. Bee. J. 139(4), 287-290. Ref. 59.

Rinderer, T.E., de Guzman, L.I., Delatte, G.T., Stelzer, J.A., Lancaster, V.A., Kuznetsov, V., Beaman, L., Watts, R., Harris, J.W., 2001. Resistance to the parasitic mite Varroa destructor in honey bees from far-eastern Russia. Apidologie 32(4), 381394. Ref. 58

Rinderer, T.E., De Guzman, L., Danka, R., 2005. A new phase begins for the USDA-ARS Russian Honey Bee breeding program. Am. Bee J. 145, 579-582.

Rinderer, T.E., Harris, J.W., Hunt, G.J., de Guzman, L.I., 2010. Breeding for resistance to Varroa destructor in North America. Apidologie 41 (3), 409-424.

Ritter, W., 1990. Development of Varroa mite population in treated and untreated colonies in Tunisia. Apidologie 21, 368-370.

Rivera-Marchand, B., Oskay, D., Giray, T., 2012. Gentle Africanized bees on an oceanic island. Evol. Appl. 5(7), 746-756. Ref. 60.

Roberts, K.E., Hughes, W.O., 2014. Immunosenescence and resistance to parasite infection in the honey bee, Apis mellifera. J. Invertebr. Pathol. 121, 1-6.

Robertson, A.J., Trost, B., Scruten, E., Robertson, T., Mostajeran, M., Connor, W. Kusalik, A., Griebel, P., Napper, S., 2014. Identification of developmentallyspecific kinotypes and mechanisms of Varroa mite resistance through wholeorganism, kinome analysis of honeybee. Front. Genet. 5, 139. Ref. 61.

Rosenkranz, P., Engels, W., 1994. Infertility of Varroa jacobsoni females after invasion into Apis mellifera worker brood as a tolerance factor against varroatosis. Apidologie. 25(4), 402-411. Ref. 62.

Rosenkranz, P., 1999. Honey bee (Apis mellifera L.) tolerance to Varroa jacobsoni Oud. in South America. Apidologie 30, 159-172.

Rosenkranz, P., Aumeier, P., Ziegelmann, B., 2010. Biology and control of Varroa destructor. J. Invertebr. Pathol. 103, 96-119.

Ruttner, F., Ritter, W., 1980. Das Eindringen von Varroa jacobsoni nach Europa im Ruckblick. Allgemeine Deutsche Imkerzeitung. 5, 130-133.

Ruttner, F., Marx, H., Marx, G., 1984. Beobachtungen über eine mögliche Anpassung von Varroa jacobsoni an Apis mellifera L. in Uruguay. Apidologie 15 (1), 43-62.

Scannapieco, A.C., Mannino, M.C., Soto, G., Palacio, M.A., Cladera, J.L., Lanzavecchia, S.B., 2017. Expression analysis of genes putatively associated with hygienic behavior in selected stocks of Apis mellifera L. from Argentina. Insectes Soc. 64 (4), 485-494. Ref. Table 3.

Schmid-Hempel, P., 2011. Ecological immunology. Evol. Parasitol., 98-140

Seeley, T.D., 1989. The honey bee colony as a superorganism. Am. Sci. 77 (6), $546-$ 553.

Seeley, T.D., 2007. Honey bees of the Arnot Forest: a population of feral colonies persisting with Varroa destructor in the northeastern United States. Apidologie. 38 (1), 19-29 Ref. 63.

Seeley, T.D., Tarpy, D.R., Griffin, S.R., Carcione, A., Delaney, D.A., 2015. A survivor population of wild colonies of European honeybees in the northeastern United States: investigating its genetic structure. Apidologie. 46(5), 654-666. Ref. 64.

Seeley, T.D., 2017. Life-history traits of wild honey bee colonies living in forests around Ithaca, NY, USA. Apidologie. 48(6), 743-754. Ref. 65.

Solignac, M., Cornuet, J.-M., Vautrin, D., Le Conte, Y., Anderson, D., Evans, J., CrosArteil, S., Navajas, M., 2005. The invasive Korea and Japan types of Varroa destructor, ectoparasitic mites of the Western honeybee (Apis mellifera), are two partly isolated clones. Proc. Biol. Sci. 272, 411-419. https://doi.org/ 10.1098/rspb.2004.2853.

Spivak, M., 1996. Honey bee hygienic behavior and defense against Varroa jacobsoni. Apidologie 27, 245-260.
Spivak, M., Reuter, G.S., 1998. Performance of hygienic honey bee colonies in a commercial apiary. Apidologie 29 (3), 291-302.

Spivak, M., Reuter, G.S., 2001. Resistance to American foulbrood disease by honey bee colonies Apis mellifera bred for hygienic behavior. Apidologie 32(6), 555565. Ref. 66.

Spötter, A., Gupta, P., Nürnenberg, G., Reinsch, N., Bienefeld, K., 2012. Development of a $44 \mathrm{~K}$ SNP assay focussing on the analysis of a varroa-specific defence behaviour in honey bees (Apis mellifera carnica). Mol. Ecol. Resour. 12, 323332. https://doi.org/10.1111/j.1755-0998.2011.03106.x.

Spötter, A., Gupta, P., Mayer, M., Reinsch, N., Bienefeld, K., 2016. Genome-wide association study of a Varroa-specific defense behavior in honeybees (Apis mellifera). J. Hered. 107 (3), 220-227.

Stanimirovic, Z., Jevrosima, S., Mirilovic, M., Stojic, V., 2008. Heritability of hygienic behavior in grey honey bees (Apis mellifera carnica). Acta Vet. (Beogr) 58 (5-6), 593-601.

Stanimirovic, Z., Jevrosima, S., Nevenka, A., Stojic, V., 2010. Heritability of grooming behavior in grey honey bees (Apis mellifera carnica). Acta Vet. (Beogr) 60 (2-3), 313-323.

Strapazzon, R., Carneiro, F.E., Guerra Jr, J.C.V., Moretto, G., 2009. Genetic characterization of the mite Varroa destructor (Acari: Varroidae) collected from honey bees Apis mellifera (Hymenoptera, Apidae) in the state of Santa Catarina, Brazil. Genet. Molec. Res. 8(3), 990-997. Ref. 67.

Strauss, U., Human, H., Gauthier, L., Crewe, R.M., Dietemann, V., Pirk, C.W., 2013. Seasonal prevalence of pathogens and parasites in the savannah honeybee (Apis mellifera scutellata). J. Invertebr. Pathol. 114(1), 45-52. Ref. 68.

Strauss, U., Dietemann, V., Human, H., Crewe, R.M., Pirk, C.W., 2016. Resistance rather than tolerance explains survival of savannah honeybees (Apis mellifera scutellata) to infestation by the parasitic mite Varroa destructor. Parasitology. 143(3), 374-387. Ref. 69.

Swanson, J.A., Torto, B., Kells, S.A., Mesce, K.A., Tumlinson, J.H., Spivak, M., 2009. Odorants that induce hygienic behavior in honeybees: identification of volatile compounds in chalkbrood-infected honeybee larvae. J. Chem. Ecol. 35 (9), $1108-1116$.

Tarpy, D.R., 2003. Genetic diversity within honeybee colonies prevents severe infections and promotes colony growth. Proceedings. Biol. Sci. 270, 99-103. https://doi.org/10.1098/rspb.2002.2199.

Tarpy, D.R., Gilley, D.C., Seeley, T.D., 2004. Levels of selection in a social insect: a review of conflict and cooperation during honey bee (Apis mellifera) queen replacement. Behav. Ecol. Sociobiol. 55 (6), 513-523.

Tarpy, D.R., Delaney, D.A., Seeley, T.D., 2015. Mating frequencies of honey bee queens (Apis mellifera L.) in a population of feral colonies in the northeastern United States. PLoS One. 10(3), e0118734. Ref. 70.

Thompson, J.N., 1994. The Coevolutionary Process. Univ. of Chicago Press, Chicago, IL, USA.

Trapp, J., McAfee, A., Foster, L.J., 2017. Genomics, transcriptomics and proteomics: enabling insights into social evolution and disease challenges for managed and wild bees. Mol. Ecol. 26 (3), 718-739.

Tsuruda, J.M., Harris, J.W., Bourgeois, L., Danka, R.G., Hunt, G.J., 2012. Highresolution linkage analyses to identify genes that influence Varroa sensitive hygiene behavior in honey bees. PLoS One. 7, e48276. Ref. 71.

Van Alphen, J.J.M., Fernhout, B., 2019. A small shift in VSH-gene frequency instead of rapid parallel evolution in bees. A comment on Oddie et al. 2018. PeerJ Preprints. 7, e27938v2.

Vandame, R., Morand, S., Colin, M.E., Belzunces, L.P., 2002. Parasitism in the social bee Apis mellifera: quantifying costs and benefits of behavioral resistance to Varroa destructor mites. Apidologie. 33(5), 433-445. Ref. 72.

Van Engelsdorp, D., Underwood, R.M., Cox-Foster, D.L., 2008. Short-term fumigation of honey bee (Hymenoptera: Apidae) colonies with formic and acetic acids for the control of Varroa destructor (Acari: Varroidae). J. Econ. Entomol. 101 (2), 256-264.

Villa, J., Danka, R., Harris, J., 2016. Selecting honeybees for worker brood that reduces the reproduction of Varroa destructor. Apidologie 47, 771-778.

Wagoner, K.M., Spivak, M., Rueppell, O., 2018. Brood affects hygienic behavior in the honey bee (Hymenoptera: Apidae). J. Econ. Entomol. 111 (6), 2520-2530.

Wagoner, K.M., Spivak, M., Hefetz, A., Reams, T., Rueppell, O., 2019. Stock-specific chemical brood signals are induced by Varroa and Deformed Wing Virus, and elicit hygienic response in the honey bee. Sci. Rep. 9, 8753.

Wallberg, A., Glémin, S., Webster, M.T., 2015. Extreme recombination frequencies shape genome variation and evolution in the honeybee Apis mellifera. PLoS Genet. 11, (4) e1005189.

Weinstock, G.M., Robinson, G.E., Gibbs, R.A., Worley, K.C., Evans, J.D., Maleszka, R., Elsik, C.G., 2006. Insights into social insects from the genome of the honeybee Apis mellifera. Nature 443 (7114), 931-949.

Wheeler, W.M., 1928. Emergent evolution and the development of societies. J. Philos. 25 (18), 502-503.

Wilfert, L., Long, G., Leggett, H.C., Schmid-Hempel, P., Butlin, R., Martin, S.J.M., Boots, M., 2016. Deformed wing virus is a recent global epidemic in honeybees driven by Varroa mites. Science 351 (6273), 594-597. 